\author{
Economics Working Paper Series
}

\author{
2018/014
}

\title{
Product Proliferation and Pricing in a Market with Positional Effects
}

George Deltas and Eleftherios Zacharias

The Department of Economics

Lancaster University Management School

Lancaster LA1 4YX

$\mathrm{UK}$ provided that full acknowledgement is given. 


\title{
Product Proliferation and Pricing in a Market With Positional Effects *
}

\author{
George Deltas $^{\dagger} \quad$ Eleftherios Zacharias ${ }^{\ddagger}$
}

June 5, 2018

\begin{abstract}
We provide an explanation for product versioning that is not driven by differential costs or consumer preference heterogeneity, and investigate its implications. Consumers care whether a product they own is better than that owned by others, and whether others own a better product than them. These positional effects can induce a firm to offer products of different quality, with the high quality product becoming more exclusive as these effects strengthen. Consumers with no positional preferences become worse off when the broader market acquires them, except following the introduction of the second product, when some such consumers become better off. Positional preference also reduce total consumer surplus holding the number of products fixed; however, they increase consumer surplus if they lead the firm to introduce a second product of sufficiently high quality. We discuss empirical implications of the theory.
\end{abstract}

J.E.L. Codes: L11, D11, D42.

Keywords: Relative consumption, status effects, positional externalities, Veblen goods.

*We would like to thank Alexei Alexandrov, Camelia Bejan, Evangelos Constantinou, Anthony Creane, Vincenzo Denicolo, Hanmming Fang, Jean J. Gabszewicz, Daniel Grodzicki, Marina Halac, Rogerio Mazali, Devin Pope, Stan Reynolds, Jean Tirole, and Hisayuki Yoshimoto for insightful discussion, and comments from seminar participants in the Lancaster Conference on Auctions and Competition, the University of Crete, the University of Macedonia, the EARIE conference, the Conference for Research in Economic Theory and Econometrics (CRETE), the International Industrial Organization Conference (IIOC, Drexel), the Midwest Economic Theory Conference, and the Greek Competition Authority. Of course, we alone are responsible for any errors contained here.

${ }^{\dagger}$ Department of Economics, University of Illinois, Urbana-Champaign, Urbana, United States (deltas@illinois.edu), and Department of Economics, Lancaster University, United Kingdom.

${ }^{\ddagger}$ Department of Economics, Athens University of Economics and Business, Athens, Greece (ezachar@aueb.gr). 


\section{INTRODUCTION}

Consumers like to "keep up with the Joneses." In fact, they often prefer to do better than the Joneses. The consumption level and product choices of others are benchmarks with which consumers often evaluate their own consumption and product choices. Owning a better product than other people can confer higher status, or simply make someone just feel better. One may also be tangibly worse off if his/her product choices are dominated by those of other consumers, as we explain later in this section. But reduced satisfaction from falling behind their peer group could simply be behavioral. Perhaps the intrinsic enjoyment that a consumer gets from a product stems from the knowledge that it is the best one around. Consumers who would be happy with the latest version of the iPhone or a tablet might be less happy purchasing it once the new version of that same gadget gets introduced. Research in behavioral economics and the economics of happiness does in fact provide support for the general idea that comparisons one makes with his or her peers do matter for his or her general happiness and welfare (see, for example, Luttmer 2005, Ferrer-i-Carbonell 2005, and Solnick and Hemenway 2005). ${ }^{1}$ Most recently, Bursztyn et. al. (2017) have performed a very convincing field experiment using credit cards that demonstrates consumers value owning a good that is better than that of other consumers (and more exclusive), and have shown that this effect is stronger when possession of the good is observable to others. ${ }^{2}$ Providing an explicit rationale for this behavioral norm is the subject of some prior theoretical literature. ${ }^{3}$ Many insights, however, can be obtained by taking such preferences as given and investigating their implications for economic choices and markets.

This paper sheds light on the implications of status concerns, or more generally "positional effects," for product pricing, product characteristics, and the number of products a firm offers to consumers. These positional effects are considered exogenous for the purpose of our analysis, their micro-foundations being the subject of behavioral microeconomics. We consider a monopolist that

\footnotetext{
${ }^{1}$ For some broader perspectives see Solnick and Hemenway (1998) and Clark, Frijters and Shields (2008).

${ }^{2}$ Recent work by Agarwal, Mikhed, and Scholnick (2018) uses lottery winners are "randomized treatment" to show that those exposed to it increase their expenditure of physical goods, but only those that are viewed by other consumers and thus subject to comparison.

${ }^{3}$ For example Bagwell and Bernheim (1996) argue that purchasing a more expensive product acts, under certain conditions, as a signal of unobserved wealth (and implicitly, ability) which is a "primitive" that individuals care about. Pesendorfer (1995) develops a model where consumers value exclusivity per se. See also Leibenstein (1950) which is perhaps the first paper to systematically consider and classify "extraneous" sources of consumer willingness to pay for a product. There is a connection between the positional effects analyzed in this paper and "conspicuous consumption" a la Veblen (1899), but there is an important distinction between them: high price for a good may enhance its signaling value (or Veblen effect), but has no such positive connotation here.
} 
sells a vertically differentiated good. The monopolist chooses whether to offer one, two, or more versions of that good. If two versions (or products) are offered, one would be the lower quality "standard" variant, while the other would the higher quality "premium" variant. If more than two versions are offered, the standard and premium variant would be complemented by a number of variants of intermediate quality. In the standard model of vertical differentiation, consumers care about product quality and product price; this remains the case in our set-up. However, in our model consumers also have positional considerations. They care about how many consumers have a product that is worse than the one they own, and how many consumers have a product that is better than the one that they own. The utility (and thus willingness to pay) for a product is higher the fewer the consumers who own a better product and the more numerous the consumers who own a worse one. Owing none of the products, i.e., not owning the good at all, is equivalent to owning a "version" that is inferior to the lowest quality product on sale.

Our framework provides an explanation for product versioning that is not driven by differential costs or by heterogeneity of consumer preferences. Indeed, to better examine the link between positional effects and product versioning or proliferation, our point of departure is a parametrization of the standard model of vertically differentiation under which a monopolist finds it optimal to produce only a single variant/product in the absence of such effects. Supply-side and other demandside drivers for product proliferation, such as differential marginal costs for higher quality products, decreasing returns, horizontal differentiation, or demand non-linearities which can lead to price discrimination and multiple product offerings are excluded from our model. ${ }^{4}$ This benchmark model is characterized by a uniform distribution of consumer willingness to pay for quality; fixed costs of product development are increasing in quality, but marginal costs are independent of it. In this environment, we characterize changes in the equilibrium induced by a strengthening of positional preferences.

In these comparative statics, we first consider the case when the monopolist optimally offers only a single product. As positional considerations become more important, the monopolist progressively charges higher prices, sells more units and earns higher profits, holding product quality fixed. But with the marginal consumer having a smaller value for quality, the returns to raising it diminish. As a result, product quality falls (sometimes sufficiently fast that product price declines rather than increases). Eventually, with further increases in positional considerations, the monopolist

\footnotetext{
${ }^{4}$ See, for example, Mussa Rosen (1978), Itoh (1983) for early relevant contributions and Besanko, Donnenfeld and White (1987) for some regulatory implications.
} 
may find it optimal to introduce a second product. At the point when the introduction of the second product becomes profitable, the two products "bracket" the quality of the single product they replace; one is of higher quality, and the other of lower quality. The high quality product becomes more "exclusive" with additional increases in positional considerations: its price goes up, and its sales volume relative to the base product decline; in contrast, the low quality product maintains the same price and its sales volume increases. Eventually, positional effects become so large that the market becomes fully covered. ${ }^{5}$

We perform standard welfare comparisons and show that, holding the number of products fixed, consumers become worse off under positional preferences. However, consumers who purchase the product will become better off when the firm introduces a second product, if that happens when positional preferences are relatively weak. ${ }^{6}$ These welfare comparisons are made across different levels of importance for positional effects; thus, they might be viewed as ill-defined. ${ }^{7}$ However, one definitely meaningful comparison is for the welfare of a small measure of consumers who do not have any positional considerations themselves, i.e., who are purely utilitarian, even as the bulk of consumers become more sensitive to the consumption choices of others. If that set of utilitarian consumers is small, it will not have an effect in the nature of the comparative statics described above. We show that these consumers become worse off as positional considerations increase in the market, as long as the number of products offered by the firm stay the same. This is because product quality does not increase, while product prices generally rise. In the one exception when prices fall (when there is a single product and the market becomes nearly covered), the accompanying quality decrease more than outweighs the reduction in prices, so that utility is lower in that case as well. However, there is one instance when some utilitarian consumers will be better off, and that is when the firm switches from selling one to selling two products. With one of the new products having a higher quality than the one that is replaced, utilitarian consumers who value quality sufficiently

\footnotetext{
${ }^{5}$ By design, the introduction of a third product is never optimal in our benchmark model. Under more general conditions, discussed in the extensions section, multiple products may be optimal. This does not detract from the insights obtained here.

${ }^{6}$ These findings echo, but also qualify, those in prior research. Some contributors to the broad literature, such as Frank $(1991,2005)$ argue that positional considerations have large negative effects on welfare and even argue for policy interventions. This appears to be true in our case when the number of products is fixed, but not necessarily in general.

${ }^{7}$ Some papers side-step this issue by making welfare comparisons holding the nature of positional preferences constant. For example, Hopkins and Kornienko (2004) consider consumers who allocate their income to the consumption of two goods, one of which is "positional." They show that the equilibrium of the consumption game between consumers depends on the distribution of income, and investigate how changes in that distribution impact different consumers.
} 
high may be better off even though they have to pay a higher price (and even if they themselves do not have positional preferences). Conversely, with the other product having lower quality but much lower price, some utilitarian consumers who hitherto were not purchasing the product will now purchase the low quality one.

Our results contrast with those of network effects, the other area of product demand where the consumption choices of all consumers affect the value of a product for each one of them. In network models, products are not vertically ranked, which makes a direct comparison with our framework somewhat difficult. However, when network effects are present, a product is more valuable the more people purchase it. A rival product may become less valuable as more consumers purchase the other product if the two networks are in competition for the provision of third party services. ${ }^{8}$ If the networks are not in competition, then the value of a product is independent of the number of consumers who purchase rival products. With positional preferences, the value of a top product is reduced if its market share is increased. The value of an intermediate (or low) ranked product increases with its market share, if its market share expands at the expense of a superior product, but it decreases with its market share if it expands at the expense of an inferior product (or the outside option). This qualitative difference in the relationship between market share and consumer willingness to pay for a product may also be useful in empirically distinguishing positional effects even in markets where other types of valuation spillover effects are present (such as network effects). ${ }^{9}$

Positional preferences and, more broadly, relative consumption, is a topic which received limited but growing attention in the Industrial Organization literature. Among the few contributions on the topic are Lambertini and Orsini $(2002,2005$, 2006) and Ghazzai (2008), which are discussed in the next section. Very recent work by Mazali and Rodrigues-Neto (2013) and Rayo (2013) forms a bridge between the IO and signaling literatures on this topic. Of direct relevance to our own work are their results that product proliferation can take place even when consumers do not have an intrinsic value for a product characteristics, if the product variant they purchase acts as a unobserved signal of a personal type. ${ }^{10}$ In this case, the product is effective a signal that consumers purchase from the firm. In our paper consumers value an intrinsic characteristic, and we are agnostic about the source

\footnotetext{
${ }^{8}$ For example, if the products are computer devices or game consoles and software developers have a limited capacity to create applications, then they may tend to focus on the larger networks and spend less effort developing applications for the smaller networks.

${ }^{9}$ Positional externalities are also distinct from congestion effects, both conceptually and in terms of implications for market equilibrium.

${ }^{10}$ Mazali and Rodrigues-Neto (2013) is one of the few contributions in this broad literature that considers potential competition, in the form of market contestability, and public regulation of status-good markets.
} 
of the positional preferences, i.e., whether they are innate or arise from signaling considerations. The latest contribution to this vein of research is Friedrichsen (2018), who has investigated the equilibrium and welfare properties of a market with consumer heterogeneity in willingness to pay for both quality and status (the complexity of the model requires heterogeneity to be limited to binary types). That model has a unique feature in that the status level associated with a good arises endogenously from the proportion of consumers who value quality among those purchasing that good, i.e., a good is confers a positive signaling value depending on who buys it.

Relative consumption and positional effects have been more widely studied in other areas of economics. The public finance literature has given some attention to relative consumption and positional effects, and in particular in their implications for taxation and public goods provision (e.g., Boskin and Sheshinski, 1978, Ljungqvist and Uhlig 2000, and Wendner and Goulder 2008). In the most recent contribution to this literature, Hopkins and Kornienko (2009) formulate a model in which consumers derive utility from a consumption good and from status (obtained based on relative consumption of a positional good). In their framework, prices are exogenous and there is a single variant of each good; consumer choice corresponds to the allocation of income to units of the each good. They focus on how changes in the distribution of income affect consumer utility, and how the government can affect welfare through taxation. Positional considerations also have implications for macroeconomics (e.g., Abel 1990 and Dupor and Liu 2003). Much of the literature emphasizes the effects of positional concerns on consumption and draws some broader conclusions (e.g., Clark and Oswald 1998, and Alpizar, Carlsson and Johansson-Stenman 2005). Somewhat unique from these research agendas is the contribution of Moldovanu, Sela, and Shi (2007) who show that positional preferences can also be used by a firm (or a society) for the design of performance-based award levels to better incentivize workers (or citizens).

It is worth noting that positional effects do not necessarily have a purely behavioral foundation. They sometimes have tangible material impact on the utility that a consumer obtains from the consumption of a good. The market for personal computers offers one important illustration. In that market, a computer becomes worse for the user in a real and tangible way if it is slower relative to the other computers. This happens because software code is optimized for the capabilities of the more recent and faster computers. ${ }^{11}$ A related explanation is that when a better product

\footnotetext{
${ }^{11}$ A computer can appear slow from a user's standpoint if it uses slow or inefficiently written software, or if it runs an operating system that strains the computer's resources. The care that software firms expend on designing efficient and fast code depends on the processing capability of the computers in the market. There is little reason to expend resources in writing fast code if computers are so fast that the gain in software efficiency results in little gain
} 
is available, consumers who would normally purchase the inferior product are no aware of more advanced features and capabilities; the more people purchase the better product, the more likely it is that other consumers will be aware of such features. Awareness of these additional possibilities can make a consumer less satisfied with a dominated product. Moreover, firms have incentives to cultivate positional effects for their products, something that we discuss in one of the extensions. Finally, exclusivity can be valued by consumers even when it is not associated with a better quality or a superior product. For example, Grilo, Shy, and Thisse (2001) provide a theoretical analysis of the market equilibrium when consumers value exclusivity in a horizontally differentiated product market. Amaldoss and Jain (2008) also belong to this class of models, broadly defined. A unique feature is the multi-period nature of their model, in which different types of consumers are considering the product in each period. The "leaders" have intrinsic value for the product, but also want to differentiate themselves from the "followers," while the "followers" have no intrinsic value for the product but want to mimic the "leaders" if the product is sufficiently popular with them. The multi-period nature of the model permits a meaningful analysis of quantity commitment by the seller, e.g., limited edition products. ${ }^{12}$

The next section of this paper outlines the model, and explains the formulation that we have chosen. It is followed by a section that solves for the market equilibrium when the monopolist has a fixed number of products to offer to the consumers. In section 4, we turn our attention to the implications for product proliferation, and solve for the equilibrium in a market where the monopolist can choose not only the prices and number of products that are offered, but also the product characteristics. We then consider, in section 5 , the welfare implications for consumers. The paper concludes with some remarks on extensions to more general specifications and on avenues for additional research on this broad agenda. All proofs are in an Appendix.

in execution time. As computers become faster, software and operating systems become more demanding. Thus, a computer that was perfectly adequate when purchased, may need to be replaced in five years because it would not be practical to run the new operating system and software on it. This is a clear positional effect. A recent paper by Deltas, Stengos, and Zacharias (2011) provides strong evidence to this effect.

${ }^{12}$ Some contributors, such as Frank (1991) and Klemperer and Padilla (1999), have extended the notion of positional externalities to the supply side. In those papers, the positional considerations of the firms are profit-based and not behavioral, but the profit effect may be derived from behavioral aspects of consumer decision making. 


\section{Model Outline and Initial Analysis}

Our model is a variation of the standard models of vertical differentiation (see Gabszewicz and Thisse 1979, Mussa and Rosen 1978, and Tirole 1988 for a textbook treatment). There is a mass of consumers, normalized to 1, with preferences for a good characterized by a single attribute. This attribute is traditionally referred to as quality, but given our focus on positional externalities, it could also be thought of as "luxuriousness." There are a number of products of this good for sale, each with a different level of quality/luxuriousness. Purchasing a product of higher quality is intrinsically valuable to each consumer, holding its price constant. Our point of departure from the benchmark model is the incorporation of an additional indirect effect, that of positional preferences. A consumer obtains additional utility from the consumption of a product that is proportional to the fraction of other consumers who own products of lesser luxuriousness or do not own the good at all (which is considered a "purchase" of a product with zero quality). Conversely, a consumer who purchases a particular product has a disutility that is proportional to the number of other consumers who purchase products of higher luxuriousness than his own. Thus, a consumer gains in utility if another consumer can no longer afford to buy the same product as his or hers and buys an inferior one; he or she loses utility if another consumer who would hitherto be purchasing the same product now purchases a superior one.

More formally, let there be $K$ products. The value of the positional attribute for product $j$ is denoted by $S_{j}$, with higher values of $j$ indicating products of higher quality, i.e., with $S_{j}>S_{k}$ if $j>k$. Each consumer $i$ is characterized by the money-equivalent value, $\theta_{i}$, he attaches to a unit increment of luxuriousness. Consumers have identical positional preferences, which we parametrize in a simple way based on the rank-order of the product he or she purchases relative to those of other consumers. In particular, consumer $i$ who purchases product $j$ at price $P_{j}$ obtains surplus of

$$
V_{i, j}=\theta_{i} S_{j}+\lambda\left(\sum_{\forall k<j} Q_{k}-\sum_{\forall k>j} Q_{k}\right)-P_{j}
$$

where $Q_{j}$ is the fraction of consumers who purchase product $j$ and $\theta$ is uniformly distributed in the interval $[0,1]$. A consumer who does not make a purchase has direct utility of zero from not owning the product but incurs positional disutility from being among those who do not own any product of the good at all. In other words, non-purchase results in utility of

$$
V_{i, 0}=-\lambda \sum_{\forall k} Q_{k}
$$


which is decreasing in the number of people who purchase any of the $K$ products on offer. It is worthwhile pointing out some additional implications of this preference structure. The utility difference between purchasing two products of adjacent quality/luxuriousness levels, i.e., from upgrading from one product to the next better one, is equal to

$$
V_{i, j+1}-V_{i, j}=\theta_{i}\left(S_{j+1}-S_{j}\right)+\lambda\left(Q_{j+1}+Q_{j}\right)-\left(P_{j+1}-P_{j}\right)
$$

In other words, positional considerations provide an additional willingness to pay for upgrading from one quality to the next that is proportional to the number of consumers who purchase either of the two products. This is because upgrading from product $j$ means that there are $Q_{j}$ consumers that you now dominate in terms of your consumption choice and $Q_{j+1}$ consumers that used to dominate you but no longer do.

This preference structure has a direct implication for the calculation of market shares conditional on prices. As is standard in vertical models, there will be a series of critical preference parameters, $\theta_{j}^{c}, j=1, \ldots, K$, with $\theta_{j+1}^{c}>\theta_{j}^{c}, \quad \forall j$ and $\theta_{j+1}^{c}=1$, such that consumers with a value of $\theta \in$ $\left[\theta_{j}^{c}, \theta_{j+1}^{c}\right]$ purchase product $j$. Then, given the uniform distribution of $\theta$, equation (3) becomes

$$
V_{i, j+1}-V_{i, j}=\theta_{i}\left(S_{j+1}-S_{j}\right)+\lambda\left(\theta_{j+2}^{c}-\theta_{j}^{c}\right)-\left(P_{j+1}-P_{j}\right)
$$

Since by definition a consumer with $\theta_{i}=\theta_{j}^{c}$ derives equal utility from purchasing product $j$ or product $j-1$ (with the 0 -th product being the null purchase for which by convention $S_{0}=0$ and $\left.P_{0}=0\right)$, when $\theta_{i}=\theta_{j}^{c}, V_{i, j}-V_{i, j-1}=0$, and equation (4) yields

$$
\theta_{j}^{c}\left(S_{j}-S_{j-1}\right)+\lambda\left(\theta_{j+1}^{c}-\theta_{j-1}^{c}\right)-\left(P_{j}-P_{j-1}\right)=0
$$

where $\theta_{0}^{c} \equiv 0$. In the absence of the positional effects, i.e., when $\lambda=0$, solving for $\theta_{j}^{c}$ yields the market share cutoffs for each product conditional on prices, which provides a straightforward way to derive the demand for each product. When positional considerations are present, however, the demand for each product is not independent of the sales of the other, i.e., the market share cutoffs cannot be solved independently even conditional on prices. Rather, they are determined simultaneously through a system of linear equations, of the form

$$
\lambda \theta_{j+1}^{c}+\left(S_{j}-S_{j-1}\right) \theta_{j}^{c}-\lambda \theta_{j-1}^{c}-\left(P_{j}-P_{j-1}\right)=0
$$

This greatly complicates the analysis. However, the special structure in our modeling framework yields sufficient tractability to fully characterize the equilibrium. 
For the special case when there is a single product on offer, the difference in utility from purchasing it and not purchasing it is given by

$$
V_{i, 1}-V_{i, 0}=\theta_{i} S_{1}+\lambda-P_{1}
$$

Notice that in this case, positional preferences are isomorphic to a uniform increase in the utility of purchasing the product. When plotting the demand for the product, an increase in positional considerations (i.e., an increase in $\lambda$ ) is equivalent to a parallel shift in its demand. This is a natural consequence of the assumption that all consumers share the same value of $\lambda$.

The preference specification and the product space are two of the differences of our work with that of Lambertini and Orsini. They consider either a monopolist who sells a single positional good (Lambertini and Orsini 2002) or two firms one of which sells a positional good and the other a standard good, with the sum of the two firms' sales being fixed (Lambertini and Orsini 2005). The second model is nearly equivalent to the first, except that the standard good (that plays the role of outside option) has utility level that is endogenously chosen by a firm. In both cases, the utility difference between purchasing and not purchasing the positional good is a function of the number of consumers who purchase the good. The reason is that, in their formulation, the consumer who does not purchase the positional good does not become worse off if more consumers purchase the positional good. ${ }^{13}$ This induces some unexpected comparative statics of the market share as positional preferences become stronger, i.e., it can either increase or decrease, whereas on our model one obtains the more natural comparative static that the market share of the positional good monotonically increases with the intensity of the positional preferences. ${ }^{14}$ Note that our work goes beyond their contribution in a number of ways, as we consider markets with more than one positional product, investigate the effect of positional preferences not only on quality but also on mark-ups, derive their effect on product introduction, and investigate the impact of positional preferences on a (few) consumers who do not have them.

The design, development, production, and marketing of each product carries a fixed cost, which

\footnotetext{
${ }^{13}$ Our preference specification is also related, but distinct, to that in Hopkins and Kornienko (2009). Unlike in our framework, they treat "ties" in the consumption of the positional good differently from strict dominance. But unlike them, we allow for different intensity in the positional preferences relative to the non-positional good. Neither specification is more general than the other. But if we introduced a difference between the disutility from being "dominated" by a set of consumers from the utility of "dominating" a set of consumers, our specification would generalize theirs.

${ }^{14}$ Ghazzai (2008) analyzes a variation of these models where the distribution of consumer types is binary.
} 
is a function of its quality. This cost is denoted by the function $\Gamma(S)$, with $\Gamma(S)^{\prime}>0, \Gamma(S)^{\prime \prime}>0$, and $\Gamma(S)^{\prime \prime \prime} \geq 0$, i.e., the cost function is not only increasing in quality and convex but also (weakly) increasingly convex. There are no cost synergies from developing a second product, i.e., if the firm sells two products of quality $S_{a}$ and $S_{b}$ the total cost is $\Gamma\left(S_{a}\right)+\Gamma\left(S_{b}\right)$. We obtain some results without further restrictions in the relationship between product quality and fixed costs, and also examine some special cases. Quality can take any value above a minimum level of $\underline{S}$. This level reflects some minimum functionality of the product and possibly also a constraint on quality imposed by the desire to maintain the image of a firm's brand. For example, when choosing the features of the iWatch, Apple was constrained by the fact that a watch must have some minimal functions (e.g., telling the time/date and having alarm and stopwatch functions). It was also implicitly constrained by the fact that an Apple smart watch had to additionally combine some of the functions of smart metering or athletic data bands. Beyond this minimal level, Apple could add further features and design elements. In section 6 we model the reputation costs that a firm's brand would suffer by introducing products below some threshold quality level and derive the (few) differences this imparts to the analysis that assumes a fixed exogenous lower bound in quality.

Producing a unit of a product of any luxuriousness has the same marginal cost, which we normalize to zero. This assumption is a reasonable approximation to reality when quality is mostly driven by $\mathrm{R} \& \mathrm{D}$ and the goods are electronic or digital (e.g., software). Importantly, this assumption ensures that in our framework, a firm has no incentives to introduce multiple products for the purpose of price discrimination in the absence of positional considerations. Thus, product proliferation arises solely due to their presence. Qualitatively similar results can be obtained if marginal costs are assumed to be increasing in quality, but these models are far messier, without necessarily being more insightful.

\section{Markets with a Fixed Number of Products}

In this section, we derive results based on the presumption that the firm produces a fixed number of products. We first derive the equilibrium when the firm offers a single product, then consider the case when the firm offers two products, and finally we investigate the possibility that the firm introduces additional products. In the next section we show how the equilibrium set of product varieties and their quality levels are jointly affected by positional preferences. 


\subsection{Single Product}

We first analyze the pricing strategy of the monopoly that produces a single product. Denote its quality by $S_{j}$, its price by $P_{j}$, the sales volume (fraction of consumers who purchase the product) by $Q_{j}$, and the profits earned, gross of fixed costs, by $\pi_{j}$. Separating the gross profits from the net profits, $\Pi_{j}$, is analytically convenient, as will become clear. For all results that are obtained for a given value of quality, i.e., for all results except for those where the quality level is determined, we will report and discuss gross profits (since development costs are sunk at that point). Equilibrium values are denoted by a star $(*)$. Dependence of equilibrium values on the value of $\lambda$ and $S_{j}$ will be made explicit when necessary and omitted when it is not, e.g., $\pi_{j}^{*}$ and $\pi_{j}^{*}\left(S_{j}, \lambda\right)$ are equivalent. Equilibrium values at the optimal value of quality with a single product are indicated with the subscript of 1 rather than $j$, i.e., $P_{1}^{*}$ rather than $P_{j}^{*}$.

Having defined the notation, we proceed to the analysis. In equilibrium, consumers with low values of $\theta$ choose not to purchase the good and consumers with high values of $\theta$ choose to purchase it, with $\theta_{j}^{c}$ the critical value of $\theta$ below which the consumers do not purchase; when the market is covered $\theta_{j}^{c}=0$. Given that $\theta_{i}$ is distributed uniformly on $[0,1], Q_{j}=1-\theta_{j}^{c}$. The following Proposition describes the product market equilibrium under the above conditions. Its proof, along with all other proofs, are in an Appendix.

Proposition 1 Suppose $\lambda<S_{j}$ and only one product is produced, with quality $S_{j}$. Then, the firm sets a price of $P_{j}^{*}=\frac{\lambda+S_{j}}{2}$, sells $Q_{j}^{*}=\frac{\lambda+S_{j}}{2 S_{j}}$ units, and earns (gross) profits $\pi_{j}^{*}=\frac{\left(\lambda+S_{j}\right)^{2}}{4 S_{j}}$. Suppose instead $\lambda \geq S_{j}$. In that case, the firm sets a price of $P_{j}^{*}=\lambda$, sells to the entire market (i.e., $Q_{j}^{*}=1$ ) and earns (gross) profits of $\pi_{j}^{*}=\lambda$.

To ascertain what is the optimal choice of quality and how it is affected by positional preferences, it is necessary to examine how the gross profit function, $\pi_{j}^{*}\left(S_{j}, \lambda\right)$, depends on $\lambda$ and $S_{j}$. We first examine the comparative statics of the market equilibrium with respect to $\lambda$, conditional on a product with quality $S_{j}$ being the only product offered. We readily obtain the following Corollary.

Corollary 1 Until the market is covered, the equilibrium price, volume, and gross profits all increase with $\lambda$ when a single product of quality $S_{j}$ is offered. When the market becomes covered, volume cannot increase further, but price and gross profits continue to increase with $\lambda$. Price, volume, and profits are all continuous in $\lambda$ when the market becomes covered. 
Intuitively, when positional effects are stronger, more consumers are willing to purchase the product at any given price. With the demand for the product shifting outwards through a parallel shift, the firm also finds it optimal to raise its price. Thus, firm profits increase. When the market becomes fully covered, further increases in positional effects permit the firm to extract more surplus from the marginal consumer, who is now the "lowest valuation" consumer and who is willing to pay more to not be left without the product.

We now look at the effect of the quality level, $S_{j}$, on gross profits, prices and sales volume. These effects are relevant for determining the optimal quality when there is a single product, and also for the comparative statics of the market equilibrium when a change in $\lambda$ induces the firm to add products. It follows by inspection that the optimal price increases with $S_{j}$ when the market is not covered, and is independent of $S_{j}$ when the market is covered. The reason for this independence when the market is covered is that the marginal consumer has (by assumption) no valuation for quality; his willingness to pay for the product arises solely from positional considerations. Hence, the price depends only on the strength of positional preferences. When the market is not covered, equilibrium sales volume decreases with $S_{j}$. This counter-intuitive response is due to the change in demand elasticity as quality increases and the fact that elasticity is a function of $\lambda$ (see Balan and Deltas 2013 for further details). ${ }^{15}$ In fact, the market is covered when product quality is low (e.g., $\left.S_{j}<\lambda\right)$ and is only partially covered when quality is high. ${ }^{16}$

The partial derivative of equilibrium gross profits with respect to $S_{j}$ when the market is partially covered is given by

$$
\begin{aligned}
\frac{\partial \pi_{j}^{*}\left(S_{j}, \lambda\right)}{\partial S_{j}} & =\frac{2\left(\lambda+S_{j}\right) 4 S_{j}-4\left(\lambda+S_{j}\right)^{2}}{16 S_{j}^{2}} \\
& =\frac{\left.\left(\lambda+S_{j}\right)\left(2 S_{j}-\lambda-S_{j}\right)\right)}{4 S_{j}^{2}}
\end{aligned}
$$

which is positive if $S_{j}-\lambda>0$, i.e., when the market is not covered.

Finally, the equilibrium price, quantity (sales volume) and gross profit are all continuous in quality when the market switches from being fully covered to being only partially covered. Substituting in the equilibrium expressions $S_{j}=\lambda$, the threshold at which the market becomes covered,

\footnotetext{
${ }^{15}$ This is one instance where assuming zero marginal cost simplifies the analysis. With a positive marginal cost, the effect of quality of market coverage would depend on whether the marginal cost is greater or lower than $\lambda$, yielding more complicated comparative statics of the market equilibrium with respect to $\lambda$ when $S_{j}$ is endogenized.

${ }^{16}$ With marginal costs being zero, the monopolist prices at the point of the demand curve were the elasticity is equal to one. When demand is linear, this point is half of the demand intercept, i.e., the optimal price is $\frac{\lambda+S_{j}}{2}$. As $S_{j}$ drops towards $\lambda$, the optimal price drops towards $\lambda$ which is the reservation utility of the lowest valuation customer when everybody else buys. The market thus becomes covered.
} 
shows that the full market coverage expressions become the same as those pertaining to partial coverage. Moreover, the slope of the profit function goes to zero as $S_{j}$ approaches $\lambda$ from above, implying that the profit function is also differentiable at that point. These findings are summarized in the corollary below.

Corollary 2 An increase in quality level $S_{j}$ has no effect on price, sales volume, and gross profits until it reaches the value of $\lambda$. For $S_{j}>\lambda$, further increases in quality increase the price and profits but result in reduced sales volume. Price, volume, and gross profits are all continuous in $S_{j}$ at $S_{j}=\lambda$, while gross profits are also differentiable at that point.

The above analysis derived the equilibrium treating product quality as fixed at an exogenous level. We now examine the choice of quality if the firm were to produce a single product. This question is relevant because in practice some firms choose to offer only a single version (or model) of their iconic products. The presence of high fixed costs at the product level may be one explanation for this, but there may be others (e.g., concerns about brand image). Some preliminary analytics will greatly facilitate the determination of this optimal quality level. Straightforward manipulation of gross profit function reported in Proposition 1 allows us to write it as

$$
\pi_{j}^{*}\left(S_{j} ; \lambda\right)=\left\{\begin{array}{lll}
\lambda & \text { if } & S_{j} \leq \lambda \\
\frac{\lambda}{2}+\frac{S_{j}}{4}+\frac{\lambda^{2}}{4 S_{j}} & \text { if } & S_{j}>\lambda
\end{array}\right.
$$

The gross profit function is flat until quality reaches $\lambda$. Thereafter, it consists of the linear term (the first two fractions in expression (9)) plus a term that is diminishing in $S_{j}$ and goes to zero as quality increases (the last fraction in expression (9)). As noted earlier, the profit function is continuous and differentiable at $\lambda$. Therefore, the gross profit function is initially flat, then increases with an increasing slope and asymptotes from above to $\frac{\lambda}{2}+\frac{S_{j}}{4}$. The optimal choice of quality maximizes the gap between gross profits and the development costs, which are given by the convex and increasing function $\Gamma\left(S_{j}\right)$. Figure 1 below plots the gross profit function, its asymptote (denoted by the set of dashes), and the development costs. It also plots the gross profit function when $\lambda=0$, which is denoted by the broken line (note that for $\lambda=0$ the gross profit function coincides with its asymptote). 


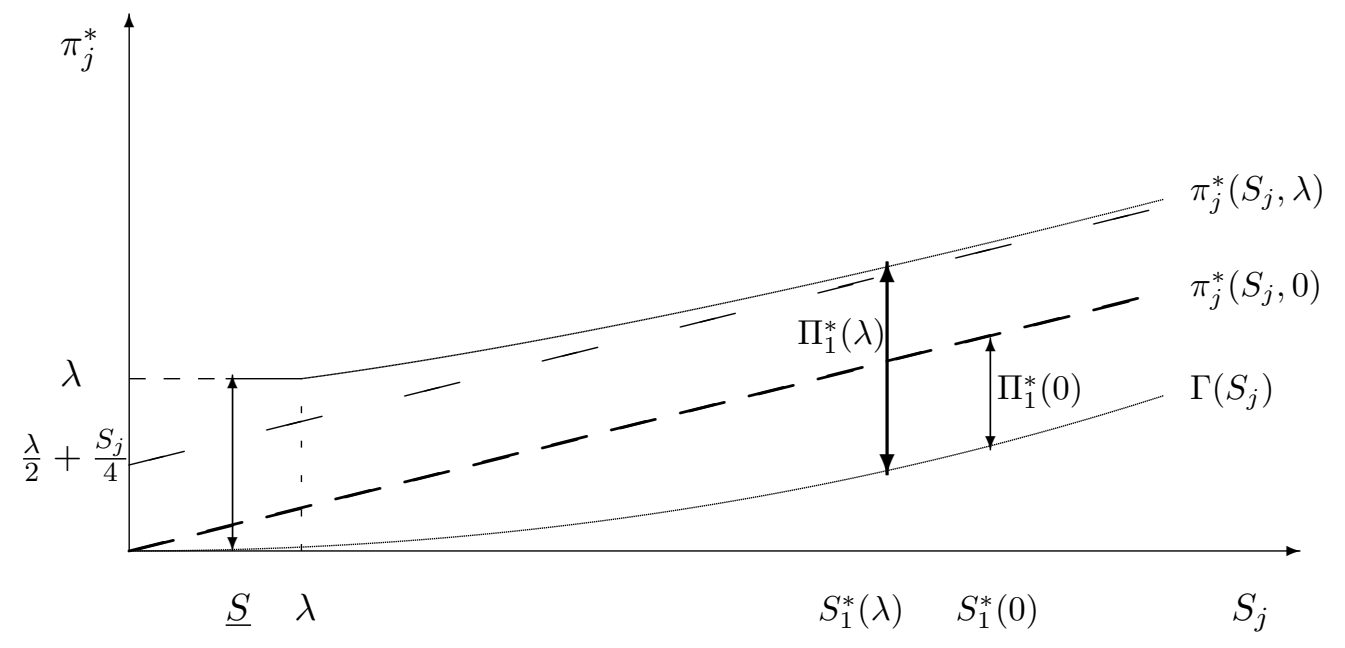

Figure 1. Profit function with a single product and optimal choice of quality.

When $\lambda=0$, (net) profits are concave in quality, being equal to the difference between the linear gross profit function and the convex cost function. There is a unique profit maximizing quality, $S_{1}^{*}(0)$, that yields profits $\Pi_{1}^{*}(0)$. These are labeled in Figure 1 . When $\lambda>0$, the gross profit's asymptote makes a parallel shift upwards, while the profit function is now located above its asymptote and consists of an initial flat segment and followed by a convex segment that is decreasingly convex (i.e., recall that $\pi^{\prime}\left(S_{j} ; \lambda\right)>0$, but observe that $\pi^{\prime \prime}\left(S_{j} ; \lambda\right)=\frac{\lambda^{2}}{2 S_{j}^{3}}>0$ while $\left.\pi^{\prime \prime \prime}\left(S_{j} ; \lambda\right)=-3 \frac{\lambda^{2}}{2 S_{j}^{4}}<0\right)$. When the value of $\lambda$ is relatively small, as drawn in Figure 1 , there are two local optima. The first is at the lowest possible quality level, $\underline{S}$ (i.e., it is a corner local optimum). The other is at the quality level where $\pi^{\prime}\left(S_{j} ; \lambda\right)=\Gamma^{\prime}\left(S_{j}\right)$ and where the second order condition of profit maximization is satisfied $\left(\pi^{\prime \prime}\left(S_{j} ; \lambda\right)-\Gamma^{\prime \prime}\left(S_{j}\right)<0\right)$. Given our assumptions on the cost function, there can be no other local optima (the proof of this and other technical statements is reserved for Proposition 2). For sufficiently small values of $\lambda$, such as that plotted in Figure 1, the second (interior) local optimum yields maximal profits. It is also apparent from the figure that $S_{1}^{*}(\lambda)<S_{1}^{*}(0)$, i.e., optimal quality declines with $\lambda$.

As the value of $\lambda$ increases, optimal quality declines further. At some point, the market becomes nearly covered, and the profit obtained by the corner solution $\left(S_{j}=\underline{S}\right)$ nearly equals that from the interior local optimum. This is shown in Figure 2 below, for $\lambda=\lambda^{\prime}$. A further increase in $\lambda$, to say $\lambda^{\prime \prime}$, results in optimal quality jumping down to $\underline{S}$ and to the market becoming fully covered. ${ }^{17}$

\footnotetext{
${ }^{17}$ Figures 1 and 2 are approximate plots of the numerical example with $\Gamma(S)=0.02 S^{2}, \lambda=1.25, \lambda^{\prime}=1.75$, $\lambda^{\prime \prime}=2.75$, and $\underline{S}=0.7$. Optimal quality levels are $S_{1}^{*}(0)=6.25, S_{1}^{*}(\lambda)=5.97, S_{1}^{*}\left(\lambda^{\prime}\right)=5.66$, and $S_{1}^{*}\left(\lambda^{\prime \prime}\right)=0.7$.
} 


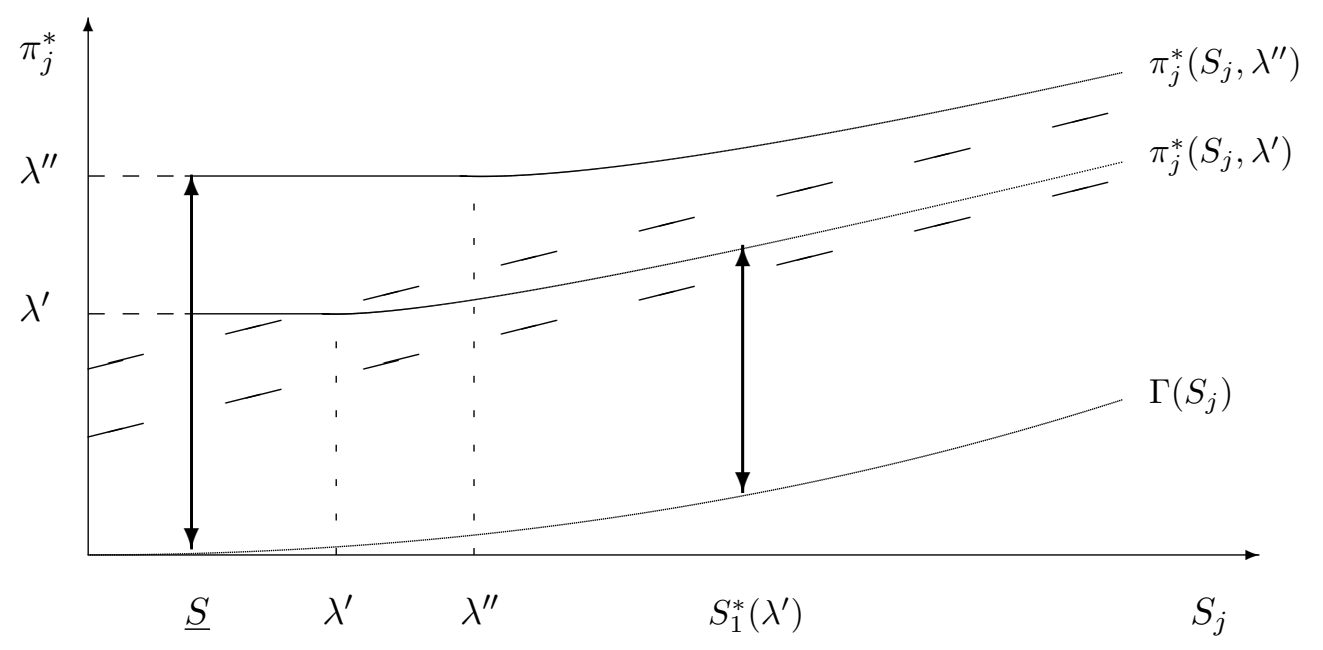

Figure 2. Profit function shifts and optimal quality changes with increases in $\lambda$.

The full comparative statics of the equilibrium when the firm produces a single product are described in the following Proposition.

Proposition 2 Optimal product quality declines smoothly with $\lambda$, until it jumps down to $\underline{S}$. Output and (net) profits are monotonically increasing in $\lambda$ (output weakly so after the market is fully covered). Finally, the effect of $\lambda$ on equilibrium price is ambiguous: increasing if optimal quality declines slowly with $\lambda$ (which is guaranteed to happen for low $\lambda$ ), but decreasing if optimal quality declines rapidly with $\lambda$ or jumps down to $\underline{S}$.

The intuition for the results in Proposition 2 is as follows. When positional considerations become stronger, part of the value of acquiring the product is its mere possession. The intrinsic quality of the product is not relevant for this component of its value. Moreover, as sales increase, the marginal consumer has a smaller value of $\theta$, i.e., values quality less. Since the firm's quality choice reflects the trade-offs at the margin of its consumer base, its incentives to invest in quality decline and thus so does its optimal quality. With the upwards shift in demand from increase in $\lambda$ and its flattening because of lower quality, optimal monopoly quantity increases. Price generally increases, but is not guaranteed to do so because the lower demand slope can lead to a reduction in its elasticity.

We conclude with a discussion of the relationship between full market coverage and optimal quality being equal to $\underline{S}$. An examination of Figures 1 and 2 reveals that when $\lambda$ increases to the point that quality jumps down to $\underline{S}$, the market may become covered; in fact, it does so in the two figures. However, this is not always the case. For values of $\underline{S}$ that exceed $\lambda$, it is possible 
that quality drops to its minimum level, while the market still remains partially covered. This is illustrated in Figure 3, which plots the optimal quality level, $S_{1}^{*}(\lambda)$, as a function of $\lambda$. The dashed line in this figure is the 45-degree line where quality is equal to $\lambda$.

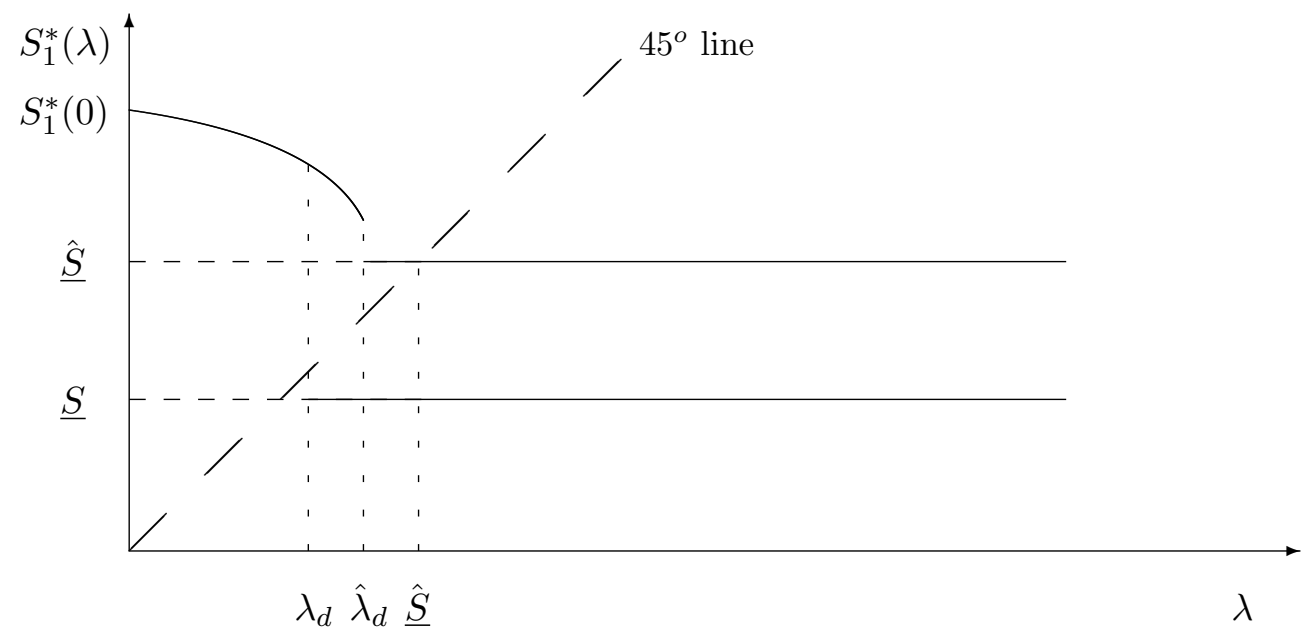

Figure 3. Market coverage and optimal quality as a function of $\lambda$ and $\underline{S}$.

In Figure 3, when the minimum quality level is equal to $\underline{S}$, optimal quality jumps down to that level at $\lambda=\lambda_{d}$. This jump results in full market coverage, since $\underline{S}<\lambda_{d}$. As $\underline{S}$ increases, but stays below $\lambda_{d}$, the profit the firm obtains from the corner solution falls, because the costs of producing quality level $\underline{S}$ increase, but there is no gain in revenue. Therefore, the value of $\lambda$ at which optimal quality jumps down to the minimum value rises, but the function $S_{1}^{*}(\lambda)$ otherwise remains the same. At some point, $\underline{S}$ exceeds the value of $\lambda$ at which quality jumps to the minimum value. When this happens, the market will not be covered, while quality will be at its minimum. This is illustrated in Figure 3 for minimum quality $\underline{\hat{S}}$, with quality dropping to the minimum value at $\lambda=\hat{\lambda}_{d}$. Optimal quality is at $\underline{\hat{S}}$ but the market is not covered for values of $\lambda \in\left[\hat{\lambda}_{d}, \underline{\hat{S}}\right]$.

\subsection{Two or More Products}

We now consider the more interesting but more complicated case of a two product firm. Let the quality levels of the two products of the firm be denoted by $S_{a}$ and $S_{b}$, with $S_{a}<S_{b}$. When $\lambda=0$ the firm offers only one, high quality product. In this "boundary" case, then, the solution is as derived in the prior section. Suppose now that $\lambda>0$. 
The market share conditions from equation (6) yield in the case of two products the system

$$
\begin{aligned}
\lambda \theta_{b}^{c}+S_{a} \theta_{a}^{c}-P_{a} & =0 \\
\lambda+\left(S_{b}-S_{a}\right) \theta_{b}^{c}-\lambda \theta_{a}^{c}-\left(P_{b}-P_{a}\right) & =0
\end{aligned}
$$

Solving this system of equations, we obtain

$$
\begin{gathered}
\theta_{a}^{c}=\frac{\lambda^{2}-S_{a} P_{a}+S_{b} P_{a}+\lambda P_{a}-\lambda P_{b}}{\lambda^{2}+S_{a} S_{b}-S_{a}^{2}} \\
\theta_{b}^{c}=\frac{S_{a} P_{b}+\lambda P_{a}-S_{a} P_{a}-\lambda S_{a}}{\lambda^{2}+S_{a} S_{b}-S_{a}^{2}} .
\end{gathered}
$$

These expressions yield the sales volume of each product as a function of prices, qualities, and $\lambda$. The results below provide the equilibrium in this market, given the quality levels.

Proposition 3 When the monopoly offers two products and the market is not covered, i.e., when $S_{a}>\lambda$, the profit maximizing prices are given by

$$
P_{a}^{*}=\frac{S_{a}}{2}
$$

and

$$
P_{b}^{*}=\frac{\lambda}{2}+\frac{\lambda^{2}}{2 S_{a}}+\frac{S_{b}}{2}
$$

the sales volume for the two products given by

$$
Q_{a}^{*}=\theta_{b}-\theta_{a}=\frac{\lambda}{2 S_{a}}
$$

and

$$
Q_{b}^{*}=1-\theta_{b}=\frac{1}{2}
$$

and gross profits by

$$
\pi_{a, b}^{*}=\frac{2 \lambda+S_{b}}{4}+\frac{\lambda^{2}}{4 S_{a}}
$$

When the market is fully covered, i.e., when $S_{a} \leq \lambda, P_{a}^{*}=\frac{\lambda}{2}, P_{b}^{*}=\frac{3 \lambda+S_{b}-S_{a}}{2}, Q_{a}^{*}=Q_{b}^{*}=\frac{1}{2}$, and $\pi_{a, b}^{*}=\frac{4 \lambda+S_{b}-S_{a}}{4}$. Moreover, all outcomes are continuous at $S_{a}=\lambda$, i.e., when the market becomes fully covered.

The comparative statics of prices, volumes, and profits with respect to $\lambda$, holding the quality levels fixed, are apparent by inspection. They are summarized in the following Corollary. 
Corollary 3 The price of the high quality product increases in $\lambda$ but its sales volume is constant. The price of the low quality product is independent of $\lambda$ but its sales volume is increasing until the market is covered; thereafter, its price is increasing in $\lambda$, but the sales volume remains constant. Profits are increasing in $\lambda$.

We now turn to the determination of the optimal quality levels when the firm produces two products. Note that the gross profit function is additive separable in the quality levels of the two products, whether or not the market is covered, so that their optimum values are determined independently. Moreover, gross profits are monotonically increasing in $S_{b}$ and monotonically decreasing in $S_{a}$, regardless of whether the market is covered or not. Then, since quality is costly, the optimal quality of the low quality product is $S_{a}^{*}=\underline{S}$. The profit maximizing quality of the high quality product when the market is not fully covered is obtained from the condition

$$
\frac{d \pi_{a, b}^{*}}{d S_{b}}-\Gamma^{\prime}\left(S_{b}^{*}\right)=0 \quad \Rightarrow \quad \frac{1}{4}-\Gamma^{\prime}\left(S_{b}^{*}\right)=0
$$

This is the same first order condition as the one for a single product when $\lambda=0$. Therefore, $S_{b}^{*}=S_{1}^{*}(0)$. The same first order condition holds when the market is covered, leading to the same value for $S_{b}^{*}$. Notice that the optimal quality of neither the low nor the high quality product depends on the value of $\lambda$. Therefore, the above corollary describes the comparative statics of the optimal price and sales volumes. The value of $\lambda$ only determines whether the market is fully covered (when $\lambda>\underline{S}$ ) or whether it is not.

We now investigate what quality levels the firm would choose if it were to offer three products, of qualities $S_{l}$ (low), $S_{m}$ (intermediate), and $S_{h}$ (high). As a first step, we derive equilibrium prices and market shares for any pre-specified level of qualities. We are able to obtain the following result.

Proposition 4 When the monopoly offers three products of different quality, the profit maximizing prices result in zero market share for the product of intermediate quality.

Given this result, the product of intermediate quality is effectively absent, which makes the three product case not relevant. ${ }^{18}$ The equilibrium would be as described in the two product case, regardless of the values of $\lambda$. It follows that the firm will not introduce four or more products.

\footnotetext{
${ }^{18}$ This result is driven by our simplifying assumptions, which keep the analysis tractable and transparent. It will not hold under more general cost and preference structures (see section on extensions).
} 


\section{Choice of the Number of Products}

We now describe how the number of products and the market equilibrium changes as $\lambda$ increases, using the results obtained in the preceding sections. We start by the observation that if $\lambda>\underline{S}$ and the market is covered, changes in $\lambda$ do not affect the optimal number of products. If the firm chooses to sell one product when $\lambda=\underline{S}$, it will also sell one product for any higher values of $\lambda$, and similarly if the firm chooses to sell two products. The reason for this is that the difference in the profits of the two product lines when the market is covered is equal to

$$
\begin{aligned}
\Pi_{2}^{*}(\lambda \mid \lambda>\underline{S})-\Pi_{1}^{*}(\lambda \mid \lambda>\underline{S}) & =\left[\lambda+\frac{S_{1}^{*}(0)-\underline{S}}{4}-\Gamma\left(S_{1}^{*}(0)\right)-\Gamma(\underline{S})\right]-[\lambda-\Gamma(\underline{S})] \\
& =\frac{S_{1}^{*}(0)-\underline{S}}{4}-\Gamma\left(S_{1}^{*}(0)\right) .
\end{aligned}
$$

This expression is independent of $\lambda$. If positive, the firm offers two products; otherwise it offers only one. The following proposition summarizes the equilibrium for values of $\lambda$ low enough that the market is not fully covered under either product line.

Proposition 5 For low values of $\lambda$, the optimal number of products is one. Profits increase slower with $\lambda$ when the firm sells one product rather than two, until the market is covered. After the market is covered profits increase at the same rate with $\lambda$ regardless of the number of products. Therefore, the firm may switch from selling one product to selling two before $\lambda$ becomes large enough that the market becomes covered, but it will not switch back to selling only one.

We provide and discuss two figures which illustrate these results (drawn and labeled with the market being fully covered when $\lambda>\underline{S}$ ). In Figure 4 , the firm offers a single product when $\lambda<\lambda_{c}$, and two products when $\lambda>\lambda_{c}$. The functions for the maximal profits conditional on the number of products, $k$, have a slope of $\frac{1}{2}$ for $\lambda=0$ which is increasing with $\lambda$ (see proof of Proposition 5). The one for $k=2$ increases at a faster rate and becomes equal to one at $\lambda=\underline{S}$, while the one for $k=1$ is less than one when $\lambda=\underline{S}$. For higher values of $\lambda$ both functions have a slope of one. 


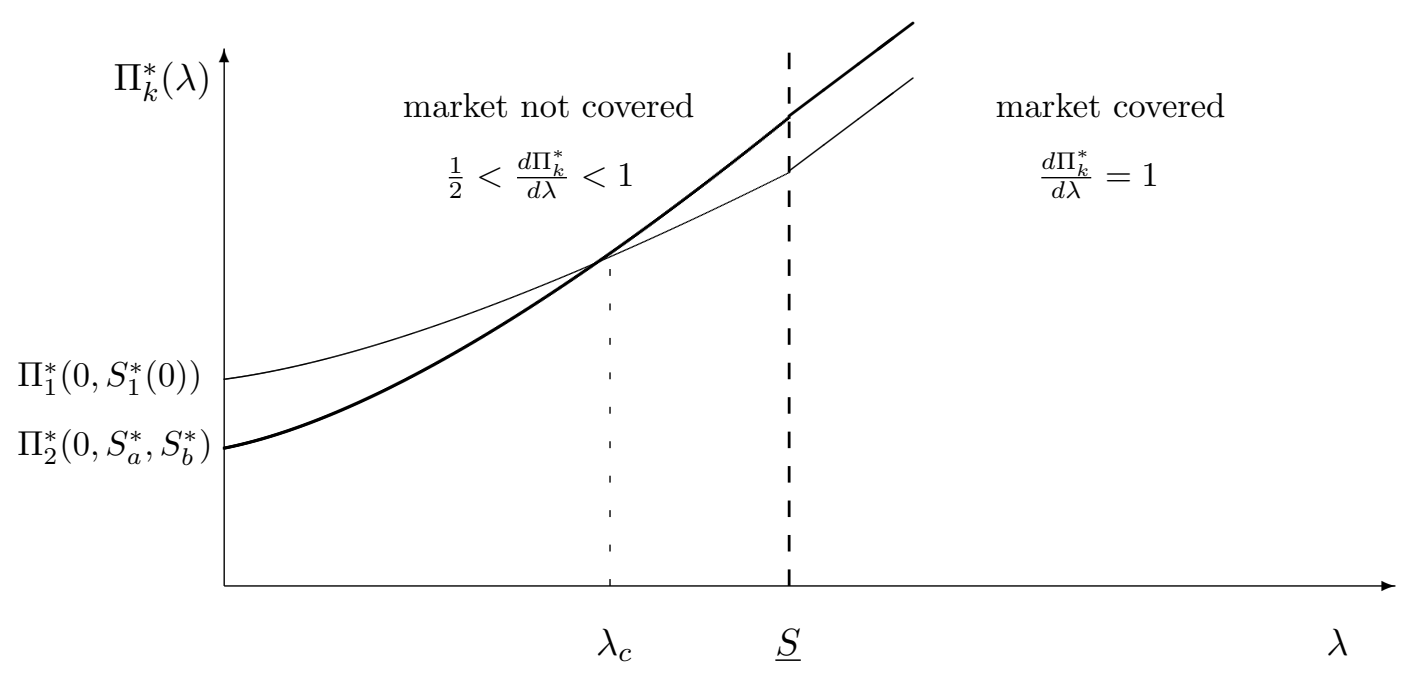

Figure 4. One or two product profits as a function of $\lambda$ : Two products optimal for $\lambda>\lambda_{c}$.

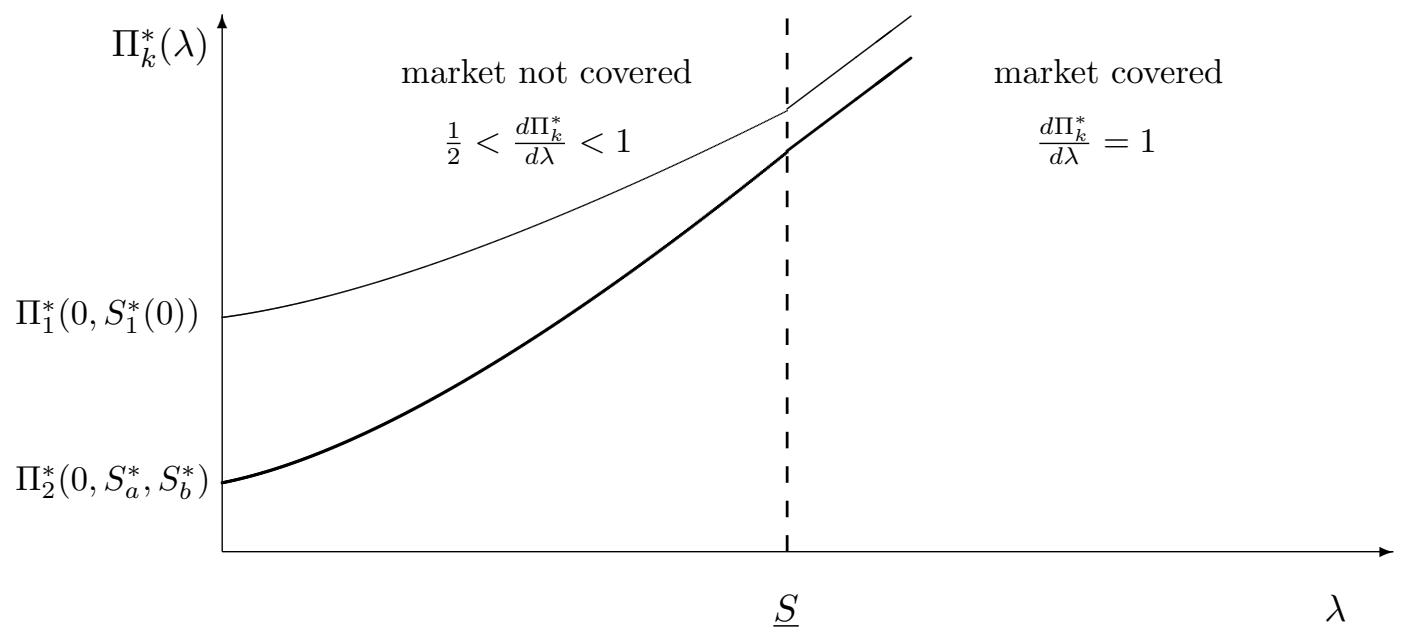

Figure 5. One or two product profits as a function of $\lambda$ : Two products never optimal.

In Figure 5, we plot an illustration for the case when the firm chooses to sell only one product regardless of the importance of the positional effects. The profit expressions have similar behavior as in Figure 4, but they never cross while the market is not covered. Therefore, they never cross even when the market becomes covered. 


\section{Welfare AnAlysis}

\subsection{Welfare Effects for Purely Utilitarian Consumers}

Does the presence of positional effects make consumers (and society) worse off? Though we do consider this question in the next section, we believe that welfare comparative statics with different preferences are fraught with difficulties of interpretation. In this section, we ask a more limited but equally interesting question. Suppose that a consumer does not have positional considerations, perhaps because he considers them frivolous, or perhaps because he belongs in a social group that does not find them important. How would such a consumer fare if the positional preferences of other consumers become more pronounced?

This turns out to be a more complicated question than it first appears. The reason is that an increase in positional considerations not only affects the optimal prices, conditional on quality, but it also affects the optimal choice of quality. It can additionally affect the number of products offered, thus providing a consumer with no positional preferences with an increased choice set, and the possibility that a product matches his or her preferences more closely. We thus first analyze the consumer surplus effects holding the number of products fixed (though allowing their quality to adjust optimally), and then investigate the effects of changing the number of products. Throughout, we assume that the mass of consumers who have no positional preferences is "epsilon" so that their presence does not affect the profit maximization calculations of the firm.

Suppose there was a single product. The utility of a "utilitarian" consumer, $R$, with no positional preferences, from purchasing that product is

$$
V_{R}=\theta_{R} S_{1}^{*}(\lambda)-P_{1}^{*}(\lambda)
$$

As the positional preferences of other consumers strengthen, quality decreases (smoothly or possibly discontinuously), which tends to decrease that consumer's utility. However, price may also decrease and in fact, does jump down to $\lambda$ when the market becomes covered. Thus, the effect of $\lambda$ on the utility of a utilitarian consumer is not immediately obvious. The next proposition, though, shows that such a consumer is, in fact, unambiguously worse off. When two products are offered, an increase in $\lambda$ does not affect their quality, but only affects their prices. In particular, when the market is not covered, it raises the price of the high quality product but has no effect on that of the low quality product, whereas when it is fully covered it raises the prices of both products. It readily follows that in this case a "utilitarian" consumer's welfare weakly declines. These results 
are stated below.

Proposition 6 A "utilitarian" consumer who purchases the product becomes worse off when the rest of the consumers have positional preferences. Moreover, his utility declines monotonically with the strength of these positional preferences effects (except when there are two products, he buys the lower quality one, and the market is not covered, in which case his utility is unaffected).

Changes in the welfare of the utilitarian consumer from marginal increases in $\lambda$ map into changes in market shares. These are worth stating separately in the proposition below to highlight some qualitative differences between the one product and the two product cases.

Proposition 7 When a single product is offered, the proportion of utilitarian consumers who purchase it monotonically declines with $\lambda$ and reaches zero when the market is covered. When two products are offered, the proportion of utilitarian consumers who purchase either one is constant in $\lambda$ until the market is covered and equal to the set of utilitarian consumers who purchase the product in the absence of positional preferences for all consumers. The proportion of utilitarian consumers who purchase the low (high) quality one monotonically increases (decreases) with $\lambda$, until it reaches one-half (zero) or the market is covered. After the market is covered, further increases in $\lambda$ decrease the proportion of utilitarian consumers who purchase either product.

One might think that since marginal strengthening of positional preferences negatively affects the welfare of utilitarian consumers in either the one or the two product case, there can be no situation that they are better off in a market with strong positional preferences relative to a market where these preferences are weak. This, however, is not the case. When the firm switches from offering one product to offering two, consumers are offered simultaneously greater variety and different prices. As a result, utilitarian consumers with relatively high or relatively low willingness to pay for quality are better off when the firm introduces two products.

Proposition 8 Among utilitarian consumers who purchase the product when there are no positional effects in the population, those with low values of $\theta$ become better off when a firm introduces a second product at $\lambda=\lambda^{\prime}$, while those with sufficiently high values of $\theta$ also become better off if $\frac{S_{1}^{*}(0)-S_{1}^{*}\left(\lambda^{\prime}\right)}{\lambda^{\prime}}>1$.

The intuition for this result is as follows. When the firm sells two rather than one products, the low quality one is sold at a much lower price. Some utilitarian consumers who have a relatively 
low willingness to pay for quality will find that product a better deal, especially since they do not mind that the product of other consumers dominates their own. But those utilitarian consumers at the other end of the spectrum may also become better off because their high valuation for quality. Recall that when the firm produces a single product, its quality declines when positional effects are more important. At some point, the firm may choose to sell two products. If at that point it did not change the quality of the high quality product, all "utilitarian" consumers would have been worse off, because its price would have increased by $\frac{\lambda^{2}}{2 \underline{S}}$, as the firm price discriminates against high valuation consumers. However, the firm finds it optimal to also increase the quality of that product. This leads to an additional increase in price, but this increase in price is only half as large as the willingness to pay of a consumer with $\theta_{R}=1$. Hence, the highest valuation consumer could become better off from the increase in the number of products if the quality increase is sufficiently large relative to the value of $\lambda$.

\subsection{Consumer Surplus Effects Inclusive of the Positional Component}

We now perform welfare analysis on the entire set of consumers, interpretational difficulties of welfare comparisons across different preferences notwithstanding. ${ }^{19}$ It is worth noting that this analysis can be of material importance in our setting only because the number of products is small. In the limit, if there were a continuum of product varieties and all consumers purchased a product, the rank-dependent component of utility would go to zero. ${ }^{20}$

It turns out the consumer surplus comparative statics inclusive of the positional component have similarities to those derived in the preceding section, which ignore the positional component. In particular, we are able to show that:

Proposition 9 All consumers become monotonically worse off when positional considerations strengthen, as long as the firm keeps the number of products the same.

Though an increase in positional considerations is bad for consumers when the number of

\footnotetext{
${ }^{19}$ One possibility to avoid these interpretational difficulties is to explicitly model the underlying causes of positional preferences, and then do comparative statics with respect to a change in the economy that would lead to a different equilibrium value for higher position.

${ }^{20}$ This is because in that case, a person's utility, excluding price, would be $\theta S+\lambda[\theta-(1-\theta)]$; the expectation of the expression in the bracket with respect to the distribution of $\theta$ is zero (we are thankful to Denicolo for making this point). Note that the utility excluding the rank-dependent component is that of a "utilitarian" consumer, which is the case we analyze in this section.
} 
products are fixed, the situation is far more nuanced when a marginal increase in $\lambda$ leads the firm to sell two rather than one product.

Proposition 10 Consumers who do not purchase any product are worse off when two are offered instead of one. However, total consumer surplus is higher with two rather than one products for low values of $\lambda$, when $\underline{S}$ is sufficiently large.

The intuition for this result is that the introduction of a second product expands the market. Those consumers who, despite the greater set of options, choose to not purchase are dominated by more consumers, and thus become worse off. Aggregate consumer surplus, can be higher if the switch to the second product happens at a low value of $\lambda$. In that case, the positive effects from increased variety outweigh the effects from higher prices, which are minimal due to the low levels of positional considerations. The condition on large value for $\underline{S}$ ensures that the increased variety does provide a product of sufficiently high intrinsic value, i.e., the additional product is sufficiently "relevant" for consumers.

\section{Extensions AND Discussion}

Endogenizing the Lower Bound of Quality. In our framework, there is a quality level that the product must meet to be marketable. One reason is that a product must have some minimum functionality to belong to a product class. For example, a smart phone must be a fully functional phone and have internet capabilities; a smart watch must be able to tell the time, and also provide minimal vital statistics and information. This provides a minimum standard that products in that category must meet. A more relevant constraint for firms with a high brand capital, which are the firms most likely to produce "status" goods, is that the product must have a level of functionality that is consistent with the brand name of the firm. The Apple iWatch must exceed in some meaningful way the basic functions of smart bands already in the market. Apple would not introduce it otherwise, without a substantial cost to its reputation. Brand considerations do not impose a strict exogenous minimum quality. Rather, the firm would trade off a slightly lower quality with a slightly larger loss in reputation capital.

Viewed in this way, a quality level below some threshold level, $S_{t h}$, would carry a reputation cost, denoted by $\Gamma_{\rho}(S)$. This cost could be low for quality levels that are right below $S_{t h}$, but would become progressively larger for further quality short-falls. Thus, $\Gamma_{\rho}(S)$ is a convex decreasing 
function that attains the value of zero at $S=S_{t h}$, with a derivative of zero at that point; the value of $\Gamma_{\rho}(S)$ remains zero for any higher values of $S$. In choosing the quality level, a firm would add this reputation cost to the fixed cost of product development, $\Gamma(S)$. This leads to only a minor modification of the analysis of the single product case. Denote the sum of the reputation and development costs by $\Gamma_{\sigma}(S)=\Gamma(S)+\Gamma_{\rho}(S)$. Then, the derivation of the optimal quality would proceed as before, with $\Gamma_{\sigma}(S)$ taking the place of $\Gamma(S)$. The sole modification is that $\Gamma_{\sigma}(S)$, while still convex, is now decreasing for sufficiently low values of $S$. Let the minimum value of $\Gamma_{\sigma}(S)$ be attained at $S=S_{\min }<S_{t h}$. Since gross profits are weakly increasing in $S$ (see Figures 1 and 2), the firm would never choose a quality level that is lower than $S_{\min }$. Because $\Gamma_{\sigma}(S)$ is qualitatively the same as $\Gamma(S)$ to the right of $S_{\text {min }}$, the analysis with endogenous lower bound of quality is the same as the one developed in this paper, but with $\underline{S}=S_{\min }$.

A slightly messier modification would take place in the two product case. As can be seen from Proposition 3, gross profits are increasing in the separation between the quality levels of the two products both when the market is partially covered and also after the market is fully covered. Because gross profits are separable in the two quality levels, and because $S_{t h}$ is certainly lower than the unconstrained high quality optimum, adding reputation costs for low quality does not affect the quality level of the higher quality product. The optimal quality of the lower quality product is, however, affected. In particular, the first order conditions of (net) profit maximization with respect to $S_{a}$ when the market is not fully covered now yield

$$
\frac{d \pi_{a, b}^{*}}{d S_{a}}-\Gamma_{\sigma}^{\prime}\left(S_{a}^{*}\right)=0 \quad \Rightarrow \quad-\frac{\lambda^{2}}{4\left(S_{a}^{*}\right)^{2}}-\Gamma_{\sigma}^{\prime}\left(S_{a}^{*}\right)=0
$$

It is immediately apparent that at $S_{a}^{*}, \Gamma_{\sigma}^{\prime}\left(S_{a}^{*}\right)<0$, i.e., that $S_{a}^{*}<S_{\min }$. Moreover, the higher the value of $\lambda$, the higher the value of $-\Gamma_{\sigma}^{\prime}\left(S_{a}^{*}\right)$ must be to satisfy the first order condition, and thus the smaller the optimal value of $S_{a}^{*}$. The value of $S_{a}^{*}$ declines until the market becomes fully covered (when $S_{a}^{*}=\lambda$ ), at which point it is given by the first order condition

$$
\frac{d \pi_{a, b}^{*}}{d S_{a}}-\Gamma_{\sigma}^{\prime}\left(S_{a}^{*}\right)=0 \quad \Rightarrow \quad-\frac{1}{4}-\Gamma_{\sigma}^{\prime}\left(S_{a}^{*}\right)=0
$$

This yields the only meaningful difference with the results derived in the earlier sections. In particular, when the quality level of the low quality product has no hard lower bound, the lower quality of the two product firm declines with $\lambda$ as long as the market is not covered and is lower than that of the single product firm when the market becomes covered. The reason for this is that the two product firm obtains a benefit from separating the quality levels of its two products that is 
absent for the single product firm. While endogenizing the lower bound in this manner is relatively straightforward, it also leads to relatively little additional gain in insight. Therefore, this case is kept separate from the main model, and presented here in the form of an extension.

Differential Marginal Costs. We have assumed that products have the same marginal cost regardless of the quality level, which we have normalized to zero. As a result, the firm would need to introduce no more that two products. It is quite possible, however, that products of higher quality are more costly to produce, and not just more costly to develop. It is well known from the literature (e.g., Mussa Rosen 1978, Itoh 1983, etc.) that in that case a firm may offer multiple quality levels of a vertically differentiated product, which complicates the analysis of the impact of positional considerations. The case of a maximum of two possible products can be analyzed with a quite general cost structure, albeit with a far greater notational load. It yields results of similar flavor to those obtained here: When consumers have positional preferences, firms raise prices (conditional on product quality), they tend to reduce quality levels (at least weakly) when the number of products is held fixed, and for some parameter values they go from selling one to selling two products (but never the reverse).

Going beyond a maximum of two products becomes rather intractable, and with no obvious gain in insight. Nonetheless, equation (3) provides some intuition on how an increase in positional considerations affects the incentives of a firm to introduce additional products. In particular, note that an increase in $\lambda$ increases the value from upgrading one quality level and thus shifts the demand functions outwards. This allows the firm to charge higher prices and earn higher profits, holding the number of products offered constant (even though aggregate sales volume may not increase, especially if the market is already covered). With the demand of all products, even notional ones, shifting outwards, incentives to introduce more products become stronger. However, balancing this is not only the fixed cost of product introduction, but also a reduction in the sales volume per product as more are offered. The latter tends to reduce the value of upgrading one quality level through a reduction of the term $Q_{j+1}+Q_{j}$. This yields an equilibrium in the number of products offered for any value of $\lambda .^{21}$ Indeed, as the sales volume of each product becomes smaller with increased product proliferation, a given increase in the value of $\lambda$ provides smaller shifts in the

\footnotetext{
${ }^{21}$ Incidentally, note that the introduction of a product of quality lower than two neighboring products will lead to a reduction in the value of upgrading between these two products, if in the new equilibrium their market shares decline. However, the introduction of a product with a quality level intermediate to two neighboring products may lead to an increase in the value of upgrading between the two hitherto neighboring products, i.e., the value of the "double" upgrade post entry may exceed the value of the single upgrade prior to entry.
} 
demand curve, and smaller incentives for further product introduction. In the case analyzed in this paper, with marginal costs being independent of quality, the maximum number of products that can be profitably introduced is only two.

Endogenous Positional Concerns. The positional preferences we have considered have been taken as exogenously given. Clearly, however, firms would benefit if consumers did compare their consumption relative to that of others, as the comparative statics in this paper show. ${ }^{22}$ It is, therefore, in their interest to expend resources to cultivate these innate tendencies of consumers. Much advertising seems designed to do exactly this. It may not fit neatly into the traditional "persuasive" versus "informative" classification that most of the literature considers. Rather, it may attempt to provide an aura of distinctiveness in a product, often relative to that same firm's other products. In fact, some well known branding distinctions seem to have this as the primary motive. The proliferation of luxury car brands such as Lexus, Infinity, and Acura, to separate the premium (and pricey) line-ups of Toyota, Nissan, and Honda from the lesser but not radically different standard models seems to be a good example of this strategy. Vikander (2010) provides formal analysis of these advertising strategies in a signaling model of status. ${ }^{23}$

Generalized Positional Effects. The framework we have developed assumes that positional effects are equally important for every consumer and ordinal, i.e., based on the quality rank order of the products. Quite clearly these are extreme cases. For example, Landis and Gladstone (2017) provide evidence that low income extraverts are more likely to spend on status goods, and thus may be more likely to plumb for a high quality product over its low quality sibling. Status considerations might also be more important for young people or other groups. In the terminology of our framework, this would suggest that $\lambda$ is not a constant, but follows a joint distribution with $\theta$. In other words, consumer heterogeneity would be reflected by a pair of draws $\left(\lambda_{i}, \theta_{i}\right)$. The forces identified in our simpler framework continue to be relevant to this more general framework, though this two dimensional extension greatly complicates the calculation of market shares and of optimal prices and quality. Two dimensional heterogeneity, however, may allow for additional product proliferation. $^{24}$

\footnotetext{
${ }^{22}$ Indeed, firms profit from positional effects even if they are unaware of their presence and price as if they were absent.

${ }^{23}$ See also Lin and Narasimhan (2006). These ideas also permeate the business community and press (see for example "Involvement and Motivational Advertising Strategies" by Alan Shaw, 2011, in Strategic-Planet.com).

${ }^{24}$ But in extreme cases, results may differ qualitatively. For example, suppose there was a deterministic relationship between $\lambda$ and $\theta$, with $\lambda^{\prime}(\theta)>0$. Then an increase in the strength of positional concerns, i.e., a upward shift and/or stretch in $\lambda(\theta)$ might lead the firm to increase quality, and to serve only higher types. This would be similar to an
} 
A more drastic departure from our framework is the possibility that the positional effects are of cardinal nature, i.e., that positional effects may depend on the quality difference between the products (see Bilancini and Boncinelli 2008). A product that is only marginally superior to another one may only confer marginally higher positional effects. If the positional effects have both an ordinal and a cardinal component, but the ordinal component is dominant, our findings would continue to hold qualitatively. However, in the extreme case when the positional effects are purely vertical, then some qualitative differences emerge. For example, in the single product case, optimal quality will be increasing rather than decreasing in $\lambda$, because stronger cardinal positional considerations are tantamount to a outward shift in the consumer willingness to pay for quality. However, the relatively small quality differences between the luxury branded and similarly equipped regularly branded vehicles mentioned earlier suggests that the ordinal component is dominant.

Empirical Content of the Theory. Does this model have any empirical implications? Is it possible to separately identify willingness to pay for quality from willingness to pay to have a rank-superior product, without relying on functional form restrictions? To meaningfully investigate this question, we need to generalize the model and allow the distribution of $\theta$ to be an arbitrary function $F(\theta)$ (the examples below implicitly also assume marginal costs are positive). It is not too difficult to see that, holding prices, qualities, and number of products fixed, for any observed outcome that arises from the combination of $\lambda>0$ and $F(\theta)$ there is exists a distribution $\tilde{F}(\theta)$ that can yield the same outcomes when $\lambda=0$. However, the two primitives yield different comparative statics, which in fact differ qualitatively, not just quantitatively. Therefore, there is no possibility that there exists a distribution $\tilde{F}(\theta)$ that yields the same outcomes as $F(\theta)$ if $\lambda$ where to change from a positive value to zero.

Though we do not make an exhaustive discussion of identification, we can demonstrate the above statement through the use of two illustrations. These demonstrate that the theory does generate meaningful restrictions to the data when we consider exogenous variations in prices (or, equivalently, quality) or when the number of products changes.

In both illustrations, we consider a market that is not fully covered and in which two products are offered for sale. For our first illustration, suppose that the firm reduces the price of the high quality product, $P_{2}$ (possibly due to a decrease in marginal costs). If $\lambda=0$, then for any distribution $\tilde{F}(\theta)$, a reduction in $P_{2}$ would not affect the number of people who do not purchase either product. upward shift in the distribution of $\theta$ itself, to which this example is isomorphic. 
In instead $\lambda>0$, then for any distribution $F(\theta)$ a reduction in $P_{2}$ increases the number of people who purchase neither product. The reason for this is that a reduction in $P_{2}$ expands the sales of the high quality product at the expense of the low quality one. Since the utility difference between buying the low quality product and buying nothing is $V_{i, 1}-V_{i, 0}=\theta_{i} S_{1}+\lambda\left(Q_{1}+Q_{0}\right)-P_{1}$, a reduction in $Q_{1}$ reduces the value of product 1 (relative to the outside option) and thus increases the number of consumers who make no purchase. Intuitively, part of the value of the low quality product to someone who buys neither is that it moves him/her up the positional pecking order. If few people buy that product, then it does not move this consumer that far up the pecking order, so it is less valuable.

For our second illustration, suppose that the firm introduces a product of intermediate quality. With a general distribution of $\theta$ and marginal costs that are increasing in quality, such an introduction could be profitable and the market share of the intermediate quality good would be positive. Let the observed market shares and critical values of $\theta$ when the firm still offers two products be rationalized by two sets of primitives: (i) $\lambda>0$ and $F(\theta)$, and (ii) (i) $\lambda=0$ and $\tilde{F}(\theta)$. Now suppose that the pricing and quality of this newly introduced product is chosen so that the sales of the low quality product are unaffected in a world where $\lambda=0$ and the distribution of $\theta$ is $\tilde{F}(\theta)$. Then, in a world with $\lambda>0$ and the distribution of $\theta$ given by $F(\theta)$, the introduction of the new product will lead to an increase in the sales of the low quality product. The reason is that the product of next higher quality above the low quality product now has a smaller market share, and is less appealing to someone with positional preferences, a consideration that is absent when $\lambda=0$. In addition, the introduction of this product also reduces the fraction of consumers who purchase none of the products (paralleling the discussion in the preceding paragraph), while under the world where $\lambda=0$ it will have no effect.

\section{Concluding Remarks}

Most consumer theory focuses on the effect of physical product attributes and branding on product demand. Demand interdependence is taken into consideration only in some cases, e.g., when it leads to network effects. This contrasts with much of the behavioral literature which shows that people compare themselves (and their consumption) with others. These comparisons affect their own consumption choices. As this paper shows, these considerations affect product pricing, and thus firm profits; and by affecting firm profits, they affect the product offerings of firms. 
The framework in this paper provides an additional explanation for price discrimination and product proliferation. Product proliferation is supported here not to cater to difference in consumer tastes but because of consumer preference for distinctiveness and exclusivity. In this paper, distinctiveness and exclusivity are of a "vertical" sort, though one can also imagine situations where it may take a different form. Similarly, differential prices and markups are supported here not from cost differences or from differences in demand elasticities for different types of consumers, but because of endogenous non-linearities in the demand that arise in equilibrium.

Empirical assessment of the presence of positional considerations is certainly feasible for markets with rapid product turnover when both old and new products are trading simultaneously. The ideas discussed at the end of the preceding section could serve as a starting point for such empirical investigation. There may also be instances where there are well identified changes in the importance of status or prestige in some markets, but not in others, leading to opportunities to test the predictions of this framework. The work of Bursztyn et al (2017) uses one such environment by comparing usage of exclusive credit cards when others observe the payer versus when they do not. ${ }^{25}$ We believe that formally exploring the quantitative importance of positional preferences and their implications for product proliferation and advertising are important possible directions to extend this of line research.

\footnotetext{
${ }^{25}$ Another possible example is if gender imbalance among singles may lead to such differentials for the gender that is in excess.
} 


\section{Appendix: Proofs of Propositions And Corollaries}

Proof of Proposition 1: We first consider the case when the market is not covered. A consumer who does not purchase the product obtains utility $V_{i, 0}=-\lambda Q_{j}$, while a consumer who purchases the product obtains a utility of $V_{i, 1}=\theta_{i} S_{j}+\lambda\left(1-Q_{j}\right)$. The value of $\theta_{j}^{c}$ (i.e., the value of $\theta_{i}$ for the consumer who obtains the same utility from buying and not buying the product, is implicitly obtained by setting $V_{i, 0}=V_{i, 1}$. Substituting in for $Q_{j}$ as a function of $\theta_{j}^{c}$, the condition becomes $-\lambda\left(1-\theta_{j}^{c}\right)=\theta_{j}^{c} S_{j}+\lambda \theta_{j}^{c}-P_{j}$ which solving for $\theta_{j}^{c}$ yields

$$
\theta_{j}^{c}=\frac{P_{j}-\lambda}{S_{j}}
$$

Given the uniform distribution of $\theta$ in the $[0,1]$ interval, the share of potential consumers who purchase the product is $Q_{j}=1-\frac{P_{j}-\lambda}{S_{j}}$. The monopoly sets $P_{j}$ to maximize its gross profits $\pi_{j}=P_{j}\left(1-\frac{P_{j}-\lambda}{S_{j}}\right)$, yielding the first order condition $1-\frac{P_{j}-\lambda}{S_{j}}-\frac{1}{S_{j}} P_{j}=0$. Solving this equation for price, we have: $P_{j}^{*}=\frac{\lambda+S_{j}}{2}$. Plugging into the expression for $\theta_{j}^{c}$ and simplifying we obtain:

$$
\theta_{j}^{c}=\frac{1}{2}-\frac{\lambda}{2 S_{j}}
$$

Given that consumers with $\theta_{i}>\theta_{j}^{c}$ purchase the product and that $\theta_{i}$ is uniformly distributed in $[0,1]$, the profit maximizing sales of the firm is $Q_{j}^{*}=\frac{\lambda+S_{j}}{2 S_{j}}$, with associated profits of $\pi_{j}^{*}=\frac{\left(\lambda+S_{j}\right)^{2}}{4 S_{j}}$.

We now turn our attention to the case when the market is covered. In that case, $P_{j}$ is such that the consumer with $\theta_{i}=0$ is indifferent between purchasing and not purchasing. If he or she where to strictly prefer to purchase, the firm would raise the price until all surplus is extracted. Since the consumer with $\theta_{i}=0$ derives no utility from product quality, the full value from purchasing is due to the positional effect. Therefore, observing equation (7), we can readily see that the price that results in zero surplus is $P_{j}=\lambda$. In fact, one equilibrium is that $S_{j}=S_{1}, P_{j}=\lambda$, and all consumers purchase. At the boundary, when the market is covered, the $Q_{j}^{*}=1, P_{j}^{*}=\lambda$ and gross profits $\pi_{j}^{*}=\lambda$.

Proof of Corollary 1: The comparative statics can easily be obtained through simple differentiation. The effect of $\lambda$ on $P_{j}^{*}$ is obtained by $\frac{\partial P_{j}^{*}(\lambda)}{\partial \lambda}=\frac{1}{2}>0$. The comparative static for the sales volume can be obtained from $\frac{\partial Q_{j}^{*}(\lambda)}{\partial \lambda}=\frac{1}{2 S_{j}}>0$. Finally, taking the derivative of profits with respect to $\lambda$ we have:

$$
\frac{\partial \pi_{j}^{*}\left(S_{j}, \lambda\right)}{\partial \lambda}=\frac{\partial\left(\frac{\left(\lambda+S_{j}\right)^{2}}{4 S_{j}}\right)}{\partial \lambda}=\frac{\lambda+S_{j}}{2 S_{j}}>0 .
$$

The proof for the case when the market is covered is apparent by inspection of the expressions in Proposition 1. The market becomes covered when $\lambda$ reaches the value of quality $S_{j}$. Continuity 
with respect to $\lambda$ at that point can be verified by substituting $S_{j}$ for $\lambda$ in the expressions for the covered and not fully covered markets, and verifying (by inspection) that they are same.

Proof of Proposition 2: We provide here the derivations for the statements that are not directly supported from the analysis in the text. We first show that the optimal interior quality (which may only be a local optimum) is unique and declines with $\lambda$. The first order condition of (net) profit function with respect to $S_{j}$, for $S_{j}>\lambda$, is

$$
\frac{d \Pi\left(S_{j} ; \lambda\right)}{d S_{j}}=0 \Rightarrow \frac{1}{4}-\frac{\lambda^{2}}{4 S_{1}^{2}}-\Gamma^{\prime}\left(S_{1}\right)=0
$$

The solution to this equation yields the interior quality optimum, indicated by $\hat{S}_{1}$, if the second order conditions are satisfied. These are given by

$$
\frac{\lambda^{2}}{2 \hat{S}_{1}^{3}}-\Gamma^{\prime \prime}\left(\hat{S}_{1}\right)<0 \quad \Rightarrow \quad \lambda^{2}-2 \hat{S}_{1}^{3} \Gamma^{\prime \prime}\left(\hat{S}_{1}\right)<0
$$

If no value of $\hat{S}_{1}>0$ satisfies the first and second order conditions, there is no interior optimum, and optimal quality is at $\underline{S}$ (it cannot be infinite because the cost function eventually crosses the gross profit function given our assumptions). Multiplying equation (28) through by $-4 \hat{S}_{1}^{2}$ and totally differentiating with respect to $\hat{S}_{1}$ and $\lambda$ we get

$$
-2 \hat{S}_{1} d \hat{S}_{1}+2 \lambda d \lambda+8 \hat{S}_{1} \Gamma^{\prime}\left(\hat{S}_{1}\right) d \hat{S}_{1}+4 \hat{S}_{1}^{2} \Gamma^{\prime \prime}\left(\hat{S}_{1}\right) d \hat{S}_{1}=0
$$

Dividing by 2 and collecting terms, we obtain

$$
\frac{d \hat{S}_{1}}{d \lambda}=\frac{\lambda}{\hat{S}_{1}-4 \hat{S}_{1} \Gamma^{\prime}\left(\hat{S}_{1}\right)-2 \hat{S}_{1}^{2} \Gamma^{\prime \prime}\left(\hat{S}_{1}\right)}
$$

This expression is negative if the denominator is negative. The first order condition (28) yields $\hat{S}_{1}-\frac{\lambda^{2}}{\hat{S}_{1}}-4 \hat{S}_{1} \Gamma^{\prime}\left(\hat{S}_{1}\right)=0 \Rightarrow \hat{S}_{1}-4 \hat{S}_{1} \Gamma^{\prime}\left(\hat{S}_{1}\right)=\frac{\lambda^{2}}{\hat{S}_{1}}$. Substituting into equation (31) we get

$$
\frac{d \hat{S}_{1}}{d \lambda}=\frac{\lambda}{\frac{\lambda^{2}}{\hat{S}_{1}}-2 \hat{S}_{1}^{2} \Gamma^{\prime \prime}\left(\hat{S}_{1}\right)}=\frac{\hat{S}_{1} \lambda}{\lambda^{2}-2 \hat{S}_{1}^{3} \Gamma^{\prime \prime}\left(\hat{S}_{1}\right)}<0
$$

The denominator is negative by the second order condition. The question remains whether there can be multiple interior optima, so that a change in $\lambda$ would potentially result in a jump from one interior optimum to another. Suppose there is another local optimum, $\tilde{S}_{1}$. Between $\hat{S}_{1}$ and $\tilde{S}_{1}$ there must a local profit minimum at, say, $\dot{S}_{1}$. At this local profit minimum, the second order conditions would require $\lambda^{2}-2 \dot{S}_{1}^{3} \Gamma^{\prime \prime}\left(\dot{S}_{1}\right)>0$. But $\Gamma^{\prime \prime}(S)>0$ by assumption, this inequality cannot be satisfied for any $\dot{S}_{1}>\min \left\{\hat{S}_{1}, \tilde{S}_{1}\right\}$, hence a second local optimum cannot exist.

We next show that the profits at the boundary increase faster with $\lambda$ than the profits at the interior optimum. The derivative of the profits at the boundary with respect to $\lambda$ is equal to 1 
(see Proposition 1 and observe that quality is held fixed). At the interior optimum (if one exists), we have $\frac{d \Pi\left(\hat{S}_{1} ; \lambda\right)}{d \lambda}=\frac{\partial \Pi\left(\hat{S}_{1} ; \lambda\right)}{\partial \lambda}+\frac{\partial \Pi\left(\hat{S}_{1} ; \lambda\right)}{\partial \hat{S}_{1}} \frac{d \hat{S}_{1}}{d \lambda}$. Since $\frac{\partial \Pi\left(\hat{S}_{1} ; \lambda\right)}{\partial \hat{S}_{1}}=0$ we have, by the envelope theorem, $\frac{d \Pi\left(\hat{S}_{1} ; \lambda\right)}{d \lambda}=\frac{\partial \Pi\left(\hat{S}_{1} ; \lambda\right)}{\partial \lambda}$. From the derivative in equation $(27)$ we can write $\frac{\partial \Pi\left(\hat{S}_{1} ; \lambda\right)}{\partial \lambda}=\frac{1}{2}+\frac{1}{2} \frac{\lambda}{\hat{S}_{1}}$. Since the market is not covered for $S_{j}>\lambda$ (and also at an interior optimum), it follows that $\frac{\partial \Pi\left(\hat{S}_{1} ; \lambda\right)}{\partial \lambda}<1$, and thus that profits at an interior optimum are less responsive to $\lambda$ than profits at the boundary.

The results we have proved to this point suffice to support the first statement of the Proposition. The result that profits are monotonically increasing in $\lambda$ is immediately obtained from the observations that, holding $S_{j}$ fixed, gross profits are monotonically increasing in $\lambda$ while costs are independent of $\lambda$. Thus, firm's profits are monotonically increasing if quality remains the same, and any change of quality will only be made if it yields an incremental increase in profits. At the interior optimum, output is given by $\frac{\lambda+\hat{S}_{1}}{2 \hat{S}_{1}}$ (see Proposition 1). This can be re-written as $\frac{1}{2} \frac{\lambda}{\hat{S}_{1}}+\frac{1}{2}$ which is immediately seen to be increasing in $\lambda$. When $\lambda$ reaches the point where the market becomes covered, output makes a jump upwards and remains constant thereafter.

The final statement of the proposition involves the effects of $\lambda$ on equilibrium price. From Proposition 1 we observe that at any interior optimum,

$$
\frac{d \hat{P}_{1}}{d \lambda}=\frac{1}{2}\left[1+\frac{d \hat{S}_{1}}{d \lambda}\right]
$$

The sign of this derivative depends on whether $\frac{d \hat{S}_{1}}{d \lambda}<-1$ or not. From the expression (32), we see that this derivative is negative, but values both smaller or bigger than minus 1 are consistent with satisfying the second order conditions. Thus, price at the interior optimum can be increasing or decreasing with $\lambda .{ }^{26}$ However, at low values of $\lambda, \frac{d \hat{S}_{1}}{d \lambda}>-1$, and in fact close to zero (the first order approximation of the denominator is $-2 \hat{S}_{1}^{3} \Gamma^{\prime \prime}\left(\hat{S}_{1}\right)$, while the first order approximation of the numerator is zero). Therefore, for low values of $\lambda$, price is increasing in $\lambda$. Moreover, when at the point the market becomes fully covered, price drops to $\lambda$ from a value that is higher than $\lambda$. This is because the slope of the gross profit function at $S_{j}=\lambda$ is zero and thus at any interior optimum, quality must be strictly higher than $\lambda$ (and thus $P_{1}^{*}>\lambda$ ). Therefore, when a marginal increase in $\lambda$ results in full market coverage and a price of $\lambda$, this is associated with a discrete drop in price.

Proof of Proposition 3: We first consider the case of $S_{a}>\lambda$ (market is not fully covered in equilibrium). Given the expressions in (12) and (13) we can write the firm's gross profit function

\footnotetext{
${ }^{26}$ An example where price is increasing in $\lambda$ is the one given in footnote 17 for the values of $\lambda$ plotted in Figures 1 and 2 (price goes from 3.125 for $\lambda=0$ to 3.61 for $\lambda=1.25$, and to 3.705 for $\lambda=1.75$. Price can be decreasing when cost function is relatively flat (i.e., when a small change in $\lambda$ reduces quality by a large amount). For example, when $\Gamma=0.04 S^{2}$ and $\underline{S}=1$, an increase of $\lambda$ from 1 to 1.05 reduces $S_{1}^{*}$ from 2.69 to 2.60 and the optimal price 1.845 to 1.825. At $\lambda=1.05, \frac{d \hat{S}_{1}}{d \lambda}=-1.6$.
} 
as

$$
\begin{gathered}
\pi_{a, b}=P_{b}\left(1-\frac{S_{a} P_{b}+\lambda P_{a}-S_{a} P_{a}-\lambda S_{a}}{\lambda^{2}+S_{a} S_{b}-S_{a}^{2}}\right)+ \\
P_{a}\left(\frac{S_{a} P_{b}+\lambda P_{a}-S_{a} P_{a}-\lambda S_{a}}{\lambda^{2}+S_{a} S_{b}-S_{a}^{2}}-\frac{\lambda^{2}-S_{a} P_{a}+S_{b} P_{a}+\lambda P_{a}-\lambda P_{b}}{\lambda^{2}+S_{a} S_{b}-S_{a}^{2}}\right) .
\end{gathered}
$$

The monopoly firm jointly sets the two prices $P_{a}$ and $P_{b}$ to maximize its gross profits (development costs are at this point sunk). Differentiating with respect to $P_{a}$ and $P_{b}$ and solving the system of first order conditions for prices we obtain:

$$
P_{a}^{*}=\frac{S_{a} S_{b}-S_{a}^{2}}{2\left(S_{b}-S_{a}\right)}
$$

which factoring out $S_{a}$ from the numerator and canceling $S_{b}-S_{a}$ from both numerator and denominator yields equation (14), and

$$
P_{b}^{*}=\frac{\lambda S_{a}+\lambda^{2}+S_{a} S_{b}}{2 S_{a}}=\frac{\lambda}{2}+\frac{\lambda^{2}+S_{a} S_{b}}{2 S_{a}}
$$

which, with a minor re-write becomes (15). Given equilibrium prices, $\theta_{b}^{*}$ and $\theta_{a}^{*}$ become

$$
\begin{aligned}
\theta_{b}^{*} & =\frac{S_{a} P_{b}+\lambda P_{a}-S_{a} P_{a}-\lambda S_{a}}{\lambda^{2}+S_{a} S_{b}-S_{a}^{2}}=\frac{S_{b}-S_{a}}{2\left(S_{b}-S_{a}\right)}, \\
\theta_{a}^{*} & =\frac{\lambda^{2}-S_{a} P_{a}+S_{b} P_{a}+\lambda P_{a}-\lambda P_{b}}{\lambda^{2}+S_{a} S_{b}-S_{a}^{2}}=\frac{S_{a}-\lambda}{2 S_{a}}
\end{aligned}
$$

Therefore, the market share of the high quality product is given by

$$
Q_{b}^{*}=1-\theta_{b}^{*}=\frac{S_{b}-S_{a}}{2\left(S_{b}-S_{a}\right)}=\frac{1}{2}
$$

The market share of the low quality product is given, after some manipulation, by

$$
Q_{a}^{*}=\theta_{b}^{*}-\theta_{a}^{*}=\frac{\lambda S_{a}-\lambda S_{b}}{2 S_{a}\left(S_{a}-S_{b}\right)}=\frac{\lambda}{2 S_{a}}
$$

Substituting from the above into the expression for gross profits and simplifying we obtain:

$$
\pi_{a, b}^{*}=\frac{S_{b}+2 \lambda}{4}+\frac{\lambda^{2}}{4 S_{a}} .
$$

We next consider the case of $S_{a} \leq \lambda$ (market is fully covered in equilibrium). If the market becomes covered when there are two products, the price of the low quality product would still be such that the consumer with zero value of $\theta$ will be indifferent between purchasing and not purchasing. In other words, appropriately modifying the expression (4) for $j=0$ (the null product), and substituting for both $\theta_{i}, \theta_{j}^{c}$, and $\theta_{j+1}$ the value of zero, we obtain

$$
\begin{aligned}
V_{i, a}-V_{i, 0} & =0\left(S_{a}-0\right)+\lambda\left(\theta_{b}^{c}-0\right)-\left(P_{a}-0\right) \Rightarrow \\
0 & =\lambda \theta_{b}^{c}-P_{a} \Rightarrow \\
P_{a} & =\lambda \theta_{b}^{c}
\end{aligned}
$$


The expression for $\theta_{b}^{c}$ can be obtained from equation (11) after substituting the $\theta_{a}^{c}=0$, and $P_{a}=\lambda \theta_{b}^{c}$, which yields

$$
\lambda+\left(S_{b}-S_{a}\right) \theta_{b}^{c}-\lambda \cdot 0-\left(P_{b}-\lambda \theta_{b}^{c}\right)=0 \quad \Rightarrow \quad \theta_{b}^{c}=\frac{P_{b}-\lambda}{a+S_{b}-S_{a}}
$$

The firm will choose price $P_{b}$ to maximize profits, given that the market shares are given by $\theta_{b}^{c}$ as derived above. With two variables: From (41) and (40) we have that the gross profits are given by

$$
\pi_{a, b}=P_{b}\left(1-\theta_{b}^{c}\right)+\lambda \theta_{b}^{c} \theta_{b}^{c}
$$

The first order condition with respect to $P_{b}$ yields $P_{b}^{*}=\frac{3 \lambda+S_{b}-S_{a}}{2}$. The gross profits at the optimum, then, are $\pi_{a, b}^{*}=\frac{4 \lambda+S_{b}-S_{a}}{4}$, and (41) and (40) yield $\theta_{b}^{c *}=\frac{1}{2}$ and $P_{a}^{*}=\frac{\lambda}{2}$. One can check that all outcomes are continuous at $S_{a}=\lambda$ (at the point the market becomes fully covered).

Proof of Proposition 4: Assume that $\lambda>0$. We first consider the case when the market is not fully covered. The market share conditions from equation (6) yield in the case of three products the system

$$
\begin{aligned}
\lambda \theta_{m}^{c}+S_{l} \theta_{l}^{c}-P_{l} & =0 \\
\left(S_{m}-S_{l}\right) \theta_{m}^{c}+\lambda\left(\theta_{h}^{c}-\theta_{l}^{c}\right)-\left(P_{m}-P_{l}\right) & =0 \\
\lambda\left(1-\theta_{m}^{c}\right)+\left(S_{h}-S_{m}\right) \theta_{h}^{c}-\left(P_{h}-P_{m}\right) & =0
\end{aligned}
$$

Notice that equation (42) is the same as in the two product case. Solving this system we obtain

$$
\begin{gathered}
\theta_{l}^{c}=\frac{-\lambda^{3}+\lambda^{2}\left(P_{l}-P_{m}+P_{h}\right)-\lambda\left(P_{l}-P_{m}\right)\left(S_{m}-S_{h}\right)+P_{l}\left(S_{l}-S_{m}\right)\left(S_{m}-S_{h}\right)}{S_{l}\left(S_{l}-S_{m}\right)\left(S_{m}-S_{h}\right)+\lambda^{2}\left(S_{l}-S_{m}+S_{h}\right)} \\
\theta_{m}^{c}=\frac{\lambda^{2} S_{l}+\left(P_{l}-P_{m}\right) S_{l}\left(S_{m}-S_{h}\right)+\lambda\left(P_{m} S_{l}-P_{h} S_{l}-P_{l} S_{m}+P_{l} S_{h}\right)}{S_{l}\left(S_{l}-S_{m}\right)\left(S_{m}-S_{h}\right)+\lambda^{2}\left(S_{l}-S_{m}+S_{h}\right)} \\
\theta_{h}^{c}=\frac{-\lambda^{3}+\lambda^{2}\left(P_{l}-P_{m}+P_{h}\right)+\left(P_{m}-P_{h}\right) S_{l}\left(S_{l}-S_{m}\right)+\lambda S_{l}\left(P_{m}-P_{l}+S_{l}-S_{m}\right)}{S_{l}\left(S_{l}-S_{m}\right)\left(S_{m}-S_{h}\right)+\lambda^{2}\left(S_{l}-S_{m}+S_{h}\right)}
\end{gathered}
$$

The expression for the profits is $\pi_{l, m, h}=P_{h}\left(1-\theta_{h}^{c}\right)+P_{m}\left(\theta_{h}^{c}-\theta_{m}^{c}\right)+P_{l}\left(\theta_{m}^{c}-\theta_{l}^{c}\right)$. By taking the first order condition with respect to $P_{l}, P_{m}$ and $P_{h}$ we have:

$$
\begin{gathered}
P_{l}^{*}=\frac{S_{l}}{2}, \\
P_{m}^{*}=\frac{\lambda^{2}+S_{l} S_{m}}{2 S_{l}}
\end{gathered}
$$

and

$$
P_{h}^{*}=\frac{\lambda S_{l}+\lambda^{2}+S_{l} S_{h}}{2 S_{l}}=\frac{\lambda}{2}+\frac{\lambda^{2}+S_{l} S_{h}}{2 S_{l}}=\frac{\lambda}{2}+\frac{\lambda^{2}}{2 S_{l}}+\frac{S_{h}}{2}
$$


the market shares are

$$
\begin{aligned}
Q_{l}^{*} & =\theta_{m}-\theta_{l}=\frac{\lambda}{2 S_{l}} \\
Q_{m}^{*} & =\theta_{h}-\theta_{m}=0 \\
Q_{h}^{*} & =1-\theta_{h}=\frac{1}{2}
\end{aligned}
$$

and the gross profits are:

$$
\pi_{l, m, h}^{*}=\frac{2 \lambda+S_{h}}{4}+\frac{\lambda^{2}}{4 S_{l}} .
$$

Therefore, the market share of the product of intermediate quality is zero, and equilibrium outcomes are the same as if this product were not offered. These derivations assumed that the market is not covered. When the market is covered, the proof proceeds analogously. In particular, as in the two products case, by substituting $\theta_{l}^{c}=0$ in (42) we have $P_{l}=\lambda \theta_{m}^{c}$. The expression for $\theta_{m}^{c}$ can be obtained after substituting $\theta_{l}^{c}=0$ and $P_{l}=\lambda \theta_{m}^{c}$ in (43) from which we obtain

$$
\theta_{m}^{c}=\frac{P_{m}-\lambda \theta_{h}^{c}}{\lambda+S_{m}-S_{l}} .
$$

The expression for $\theta_{h}^{c}$ can similarly be obtained from (44) from which we get

$$
\theta_{h}^{c}=\frac{\lambda \theta_{m}^{c}+P_{h}-P_{m}-\lambda}{S_{h}-S_{m}}
$$

By solving (51) and (52) we have:

$$
\theta_{m}^{c}=\frac{\lambda^{2}+\lambda\left(P_{m}-P_{h}\right)+P_{m}\left(S_{h}-S_{m}\right)}{\lambda^{2}+\left(S_{m}-S_{l}\right)\left(S_{h}-S_{m}\right)+\lambda\left(S_{h}-S_{m}\right)}
$$

and

$$
\theta_{h}^{c}=\frac{-\lambda^{2}+\left(P_{h}-P_{m}\right)\left(S_{m}-S_{l}\right)+\lambda\left(P_{h}-S_{m}+S_{l}\right)}{\lambda^{2}+\left(S_{m}-S_{l}\right)\left(S_{h}-S_{m}\right)+\lambda\left(S_{h}-S_{m}\right)}
$$

The expression for the profits in the covered case, then, is $\pi_{l, m, h}=P_{h}\left(1-\theta_{h}^{c}\right)+P_{m}\left(\theta_{h}^{c}-\theta_{m}^{c}\right)+\lambda \theta_{m}^{c} \theta_{m}^{c}$. By taking the first order condition with respect to $P_{h}$ and $P_{m}$, we have

$$
\begin{aligned}
P_{m}^{*} & =\frac{2 \lambda-S_{l}+S_{m}}{2}, \\
P_{h}^{*} & =\frac{3 \lambda-S_{l}+S_{h}}{2},
\end{aligned}
$$

and $\theta_{h}^{c}=\frac{1}{2}, \theta_{m}^{c}=\frac{1}{2}$ and $P_{l}=\frac{\lambda}{2}$. The profits are

$$
\pi_{l, m, h}^{*}=\frac{4 \lambda+S_{h}-S_{l}}{4}
$$

Note that the above are the same as with the partially covered market when we set $S_{l}=\lambda$.

Proof of Proposition 5: When the market is not covered and the firm is selling two products, their quality and the associated costs are independent of $\lambda$. Thus, $\frac{d \Pi_{2}^{*}(\lambda)}{d \lambda}=\frac{1}{2}+\frac{1}{2} \frac{\lambda}{\underline{S}}$. When the firm 
is selling only one product, we have $\frac{d \Pi_{1}^{*}\left(\lambda, S_{1}^{*}(\lambda)\right)}{d \lambda}=\frac{\partial \Pi_{1}^{*}}{\partial \lambda}+\frac{\partial \Pi_{1}^{*}}{\partial S_{1}^{*}} \frac{d S_{1}^{*}}{d \lambda}$. But by the envelope theorem $\frac{\partial \Pi_{1}^{*}}{\partial S_{1}^{*}}=0$ and therefore $\frac{d \Pi_{1}^{*}\left(\lambda, S_{1}^{*}(\lambda)\right)}{d \lambda}=\frac{\partial \Pi_{1}^{*}}{\partial \lambda}=\frac{1}{2}+\frac{1}{2} \frac{\lambda}{S_{1}^{*}(\lambda)}$. Since $S_{1}^{*}(\lambda)>\underline{S}$ when the market is not covered, $\frac{d \Pi_{2}^{*}(\lambda)}{d \lambda}>\frac{d \Pi_{1}^{*}(\lambda)}{d \lambda}$. When $\lambda=0$, the firm produces a single product (since the second product adds costs but no revenue), but as $\lambda$ increases, the difference between $\Pi_{2}^{*}(\lambda)$ and $\Pi_{1}^{*}(\lambda)$ decreases. This does not guarantee that the two profit functions will cross before the market is covered, but they may. However, it guarantees that if the firm adds the second product it will never return to sell only one as $\lambda$ continues to increase.

The last remaining possibility is that the market is covered when the firm offers one product, but not when it offers two, or vice versa. When the firm offers two products, the market becomes covered as soon as $\lambda$ reaches $\underline{S}$. By contrast, when the firm offers one product, the market may not be covered even if $\lambda$ exceeds $\underline{S}$, as can be seen from the lower of the two $S_{1}^{*}(\lambda)$ lines in Figure 3 . However, when $\lambda<\underline{S}$ neither market is fully covered. Therefore, if the market is covered when the firm offers a single product, it must be covered when the firm offers two, but the converse is not necessarily true. Suppose, then, that for some value of $\lambda$ the market is covered when the firm offers two products but not covered if it offers only one. What is the possible effect of an increase in $\lambda$ on the product line choice? During this range of $\lambda$, profits from offering two products increase faster than profits from selling one (the derivative of the former with respect to $\lambda$ is equal to 1 , while the derivative of the other is less than 1). If the firm has already switched from selling one product to selling two, it will continue to sell two products. However, if the firm offers only a single product, it may add a second.

Proof of Proposition 6: (a) One Product for Sale: If the market is not fully covered, then substituting for the optimal price as a function of optimal quality yields

$$
\begin{aligned}
V_{R} & =\theta_{R} S_{1}^{*}-\frac{\lambda+S_{1}^{*}}{2} \\
& =\frac{\left(2 \theta_{R}-1\right) S_{1}^{*}-\lambda}{2}
\end{aligned}
$$

Clearly, holding product quality constant, an increase in $\lambda$ reduces that consumer's utility. But quality also decreases with $\lambda$, so the total effect potentially depends on the sign of $2 \theta_{R}-1$. Note that when $\lambda=0, P_{1}^{*}=\frac{S_{1}^{*}}{2}$, and the consumer will purchase the good if $\theta_{R} S_{1}^{*}-\frac{S_{1}^{*}}{2}>0 \Rightarrow \theta_{R}>\frac{1}{2}$. Therefore, $2 \theta_{R}-1>0$ for any consumer who would have bought the product in the absence of positional effects in the market. It follows that if this consumer is not subject to positional considerations, an increase in $\lambda$ leaves him or her unambiguously worse off, if the market remains partially covered. Moreover, this reduction in utility is monotonic since optimal quality declines monotonically with $\lambda$. Utility also declines if optimal quality jumps down from some level $S^{\prime}$ to $\underline{S}$, because the function that gives the optimal price conditional on $S$ is continuous in quality, and thus the magnitude of the associated price jump is equal to the integral of $\frac{d P^{*}(S)}{d S}$ between $S^{\prime}$ and $\underline{S}$. 
(b) Two Products for Sale: The (weakly) monotonic decline in utility from an increase in $\lambda$ follows from the discussion preceding the proposition.

Proof of Proposition 7: (a) One Product for Sale: Let the value of $\theta_{R}$ for a utilitarian consumer who is indifferent between purchasing and not purchasing the product be $\theta_{R}^{c}$. From equation (54), this value satisfies $\left(2 \theta_{R}^{c}-1\right) S_{1}^{*}-\lambda=0$, or $\theta_{R}^{c}=\frac{1}{2}+\frac{\lambda}{2 S_{1}^{*}(\lambda)}$. This is increasing in $\lambda$ since $S_{1}^{*}(\lambda)$ is decreasing in $\lambda$. When the market (just) becomes covered, $\lambda=\underline{S}$, and $\theta_{R}^{c}$ becomes equal to one. Therefore no utilitarian consumer will purchase the product, since the distribution of $\theta$ has a support that was normalized on the unit interval.

(b) Two Products for Sale: When the market is not covered, the price of the low quality product is $P(\underline{S})=\frac{S}{2}$. The utility of a utilitarian consumer from purchasing this product is $V_{R}^{a}=\theta_{R} \underline{S}-\frac{S}{2}$, independent of $\lambda$, and positive for $\theta_{R}>\frac{1}{2}$. The utility of a utilitarian consumer from purchasing the high quality product is $V_{R}^{b}=\theta_{R} S_{b}^{*}-P_{b}^{*}$ which is equal to $V_{R}^{b}=\theta_{R} S_{b}^{*}-\frac{\lambda}{2}-\frac{\lambda^{2}}{2 S}-\frac{S_{b}^{*}}{2}$. The utilitarian consumer will prefer the high quality product over the low quality one if $\theta_{R} S_{b}^{*}-\frac{\lambda}{2}-\frac{\lambda^{2}}{2 S}-\frac{S_{b}^{*}}{2}>\theta_{R} \underline{S}-\frac{S}{2}$ i.e., for $\theta_{R}>\frac{1}{2}+\frac{\lambda}{2\left(S_{b}^{*}-\underline{S}\right)}+\frac{\lambda^{2}}{2 \underline{S}\left(S_{b}^{*}-\underline{S}\right)}$. The RHS is equal to $\frac{1}{2}$ when $\lambda=0$ and is increasing in $\lambda .{ }^{27}$ After the market is covered, the prices of both products increase, with $P_{b}^{*}$ increasing at three times the rate of $P_{a}^{*}$. The combined share declines, as does the relative share of the high quality product.

Proof of Proposition 8: We only need to consider the case of markets that are not fully covered, since if the firm introduces a second product it will do so only when the markets are partially covered. From Proposition 7 we know that when for any $\lambda^{\prime}>0$, the critical value of $\theta_{R}^{c 1}$, such that a utilitarian consumer is indifferent between purchasing and not purchasing exceeds $\frac{1}{2}$. When the firm produces two products, rather than one, the utilitarian consumers with $\theta \in\left[\frac{1}{2}, \theta_{R}^{c 1}\right.$ will switch from purchasing nothing to purchasing the low quality product. These consumers will be better off (by continuity, so will be some consumers with values of $\theta$ that are somewhat higher than $\theta_{R}^{c 1}$, since their utility when there is only one product for sale is close to zero, while their utility from buying the low quality product is strictly higher than zero).

Let us know focus on utilitarian consumers with high values of $\theta$. When the firm offers two products, the quality of the better one is $S_{1}^{*}(0)$. At the time of switch from one to two products, the utility of a "utilitarian" consumer who purchases the (single available) product is $U_{1}\left(\theta_{R}\right)=$ $\theta_{R} S_{1}^{*}\left(\lambda^{\prime}\right)-\frac{\lambda^{\prime}+S_{1}^{*}\left(\lambda^{\prime}\right)}{2}$. Right after the second product is introduced, the utility changes to $U_{2}\left(\theta_{R}\right)=$

\footnotetext{
${ }^{27}$ It is possible that it reaches unity before the market is covered, which would imply that every utilitarian consumers purchases the low quality product. Note that when the market (just) becomes covered (at what point $\lambda=\underline{S}$ ), it is equal to $\frac{1}{2}+\frac{\underline{S}}{2\left(S_{b}^{*}-\underline{S}\right)}+\frac{\underline{S}}{2\left(S_{b}^{*}-\underline{S}\right)}=\frac{1}{2}+\frac{\underline{S}}{\left(S_{b}^{*}-\underline{S}\right)}$. For $\underline{S}$ high enough, this would exceed unity.
} 
$\theta_{R} S_{1}^{*}(0)-\frac{\lambda^{\prime}+S_{1}^{*}(0)}{2}-\frac{\lambda^{\prime 2}}{2 \underline{S}}$. The utility increases when the second product is introduced if

$$
\begin{gathered}
\theta_{R} S_{1}^{*}(0)-\frac{\lambda^{\prime}+S_{1}^{*}(0)}{2}-\frac{\lambda^{\prime 2}}{2 \underline{S}}>\theta_{R} S_{1}^{*}\left(\lambda^{\prime}\right)-\frac{\lambda^{\prime}+S_{1}^{*}\left(\lambda^{\prime}\right)}{2} \Rightarrow \\
\theta_{R}\left(S_{1}^{*}(0)-S_{1}^{*}\left(\lambda^{\prime}\right)\right)-\frac{S_{1}^{*}(0)}{2}-\frac{\lambda^{\prime 2}}{2 \underline{S}}>-\frac{S_{1}^{*}\left(\lambda^{\prime}\right)}{2}
\end{gathered}
$$

Note that the left hand side is increasing in $\theta_{R}$ while the right hand side is independent of $\theta_{R}$. Thus, if this inequality is not satisfied for $\theta_{R}=1$, it will not be satisfied for any $\theta_{R}$. This allows us to write the above inequality as

$$
\begin{gathered}
\frac{S_{1}^{*}(0)}{2}-\frac{\lambda^{\prime 2}}{2 \underline{S}}>\frac{S_{1}^{*}\left(\lambda^{\prime}\right)}{2} \Rightarrow \\
S_{1}^{*}(0)-S_{1}^{*}\left(\lambda^{\prime}\right)>\frac{\lambda^{\prime 2}}{\underline{S}}
\end{gathered}
$$

If the market is not covered, it must be that $\lambda^{\prime}<\underline{S}$. Thus, a sufficient condition for some utilitarian consumers to benefit from the introduction of the second product is $S_{1}^{*}(0)-S_{1}^{*}\left(\lambda^{\prime}\right)>\lambda^{\prime}$, i.e., that the quality of single product declines sufficiently steeply with a strengthening of the positional considerations.

Proof of Proposition 9: Single Product Case: We drop the product subscript $j$ to simplify notation. Suppose the market is not fully covered. Because the distribution of $\theta$ is uniform and utility of consumers who purchase the product is linear in $S$, the mean utility of consumer a consumer who purchases the product is the average of the utility of the consumer who is indifferent between purchasing and the utility of the consumer with $\theta=1$. The utility of the consumer who is indifferent is $U\left(\theta^{c}\right)=\theta^{c} S+\lambda Q-P$. Substituting the expressions from Proposition 1 and equation (26) and simplifying, we obtain $U\left(\theta^{c}\right)=-\frac{-\lambda(\lambda+S)}{2 S}$. The utility of a consumer with $\theta=1$ is given by $U(1)=\frac{S^{2}-\lambda^{2}}{2 S}$. Taking the average of these utilities and multiplying by the fraction of consumer who purchase, $Q$, we obtain the consumer surplus of the buyers, $C S^{b}=-\frac{(2 \lambda-S)(\lambda+S)^{2}}{8 S^{2}}$. All consumers who do not buy have the same (negative) utility, which is independent of the value of $\theta$. Each non-buyer's utility is the same as that of the critical purchasing consumer. Multiplying this value by the fraction of consumers who do not purchase, we obtain $C S^{n}=\frac{\lambda(\lambda-S)(\lambda+S)}{4 S^{2}}$. Summing up $C S^{b}$ and $C S^{n}$ and simplifying we obtain $C S=\frac{-3 \lambda^{2}-2 \lambda S+S^{2}}{8 S}$. This is clearly decreasing in $\lambda$.

Suppose the market is covered. Then further increases in $\lambda$ raise $P$ but leave $S$ unaffected, thereby decreasing $C S$. Thus, consumer surplus declines with $\lambda$ when a single product is offered.

Two Product Case: Suppose the market is not covered. The utility of the consumer who is indifferent between purchasing the high and low quality products is given by $U\left(\theta_{b}^{*}\right)=\theta_{b}^{*} S_{b}+\lambda(1-$ $\left.Q_{b}^{*}\right)-P_{b}^{*}$. Substituting in from Proposition 3 , recalling that $\theta_{b}^{*}=\frac{1}{2}$, and simplifying we obtain $U\left(\theta_{b}^{*}\right)=-\frac{\lambda^{2}}{2 S_{a}}$. The utility of a consumer with $\theta=1$ is similarly obtained to be $U(1)=-\frac{\lambda^{2}-S_{a} S_{b}}{2 S_{a}}$. Applying the reasoning in the single product case part of the proof, the consumer surplus of those who purchase the high quality product is equal to the number of people who purchase it (equal 
to $\frac{1}{2}$ ), times the average of these utilities, which yields $C S^{h}=\frac{S_{a} S_{b}-2 \lambda^{2}}{8 S_{a}}$. Next, the utility of the consumer with $\theta=\theta_{a}^{*}$, who is indifferent between purchasing the low quality good and purchasing nothing is equal to $U\left(\theta_{a}^{*}\right)=\theta_{a}^{*} S_{a}+\lambda\left(\theta_{a}^{*}-Q_{b}^{*}\right)-P_{a}^{*}$. Substituting expressions from Proposition 3 and from equation (35), we obtain $U\left(\theta_{a}^{*}\right)=-\frac{\lambda^{2}}{2 S_{a}}$. The consumer surplus of those purchase the low quality product is equal to $Q_{a}^{*}$ times the average of $U\left(\theta_{a}^{*}\right)$ and $U\left(\theta_{b}^{*}\right)$. We, therefore, obtain after some manipulations, $C S^{l}=-\frac{\lambda^{2}\left(2 \alpha+S_{a}\right)}{8 S_{a}^{2}}$. The utility of those who do not purchase is equal to $U\left(\theta_{a}^{*}\right)$, and independent of $\theta$. The consumer surplus of those who do not buy is $\theta_{a}^{*}$ times $U\left(\theta_{a}^{*}\right)$, or $U^{n}=\frac{\lambda\left(\lambda-S_{a}\right)\left(\lambda+S_{a}\right)}{4 S_{a}^{2}}$. The consumer surplus of all consumers, $C S^{h}+C S^{l}+C S^{n}$ is obtained, after some manipulations, to be $C S=\frac{-3 \lambda^{2}-2 \lambda S_{a}+S_{a} S_{2}}{8 S_{a}}$. It is readily seen that $C S$ is decreasing in $\lambda$. Suppose the market is covered. Then, further increases in $\lambda$ increase both prices, but qualities and sales are unaffected. Thus, consumer surplus declines with $\lambda$ when two products are offered.

Proof of Proposition 10: Recall from the proof of Proposition 10, that the utility of those who do not buy at all when there is one product is $-\frac{\lambda(\lambda+S)}{2 S}$. Those who don't purchase when two products are available have utility $-\frac{\lambda\left(\lambda+S_{a}\right)}{2 S_{a}}$. Since the ratio in these expressions is declining in the level of product quality, and since $S>S_{a}$ at the same value of $\lambda$, the utility of those who do not buy is smaller when there are two variants.

We now look at aggregate consumer surplus. When there is one product, $C S_{1}=\frac{-3 \lambda^{2}-2 \lambda S+S^{2}}{8 S}$, while when there are two products, $C S_{2}=\frac{-3 \lambda^{2}-2 \lambda S_{a}+S_{a} S_{b}}{8 S_{a}}$. Consumers are in aggregate better off when a second product is introduced, holding $\lambda$ (nearly) constant, if

$$
\begin{aligned}
\frac{-3 \lambda^{2}-2 \lambda S+S^{2}}{8 S} & <\frac{-3 \lambda^{2}-2 \lambda S_{a}+S_{a} S_{b}}{8 S_{a}} \Rightarrow \\
3 \lambda^{2}\left(S-S_{a}\right) & <S_{a} S\left(S_{b}-S\right) \Rightarrow \\
3 \frac{\lambda^{2}}{S_{b}-S} & <\frac{S_{a} S}{S-S_{a}} \Rightarrow \\
3 \frac{\lambda^{2}}{S_{b}-S} & <\frac{S}{\frac{S}{S_{a}}-1} .
\end{aligned}
$$

The fraction on the right hand side goes to infinity as $\underline{S}$ increases towards $S$ (recall that $S_{a}=\underline{S}$ ). Both the numerator and the denominator of the fraction on the left hand side go to zero as $\lambda$ goes to zero (recall that if the firm introduces a second product at a low value of $\lambda, S$ will be close to $\left.S_{b}\right)$. Using L'Hopital's rule, the fraction becomes $-(2 \lambda) /(d S / d \lambda)$. But we know from inequality (32) that the limit of the denominator is still zero as $\lambda$ goes to zero. Taking L'Hopital's rule again, and evaluating at $\lambda=0$ yields a numerator of -2 and a denominator of $-\frac{1}{2 S^{2} \Gamma^{\prime \prime}(\cdot)}$. Thus, the limit is $2 S^{2} \Gamma^{\prime \prime}(\cdot)$, a finite positive number. Therefore, the above inequality holds for sufficiently small values of $\lambda$ when $\underline{S}$ is large enough. 


\section{REFERENCES}

Abel, A.B. (1990), "Asset Prices under Habit Formation and Catching up with the Joneses," American Economic Review Papers and Proceedings vol. 80, pages 38-42.

Agarwal, S., V. Mikhed, and B. Scholnick (2018), "Does the Relative Income of Peers Cause Financial Distress? Evidence from Lottery Winners and Neighboring Bankruptcies," Federal Reserve Bank of Philadelphia Working Paper 18-16.

Alpizar, F., F. Carlsson, and O. Johansson-Stenman (2005), "How Much do we Care About Absolute versus Relative Income and Consumption," Journal of Economic Behavior and Organization vol. 56, pages 405-421.

Amaldoss, W., and S. Jain (2008), "Trading Up: A Strategic Analysis of Reference Group Effects," Marketing Science, vol. 27, pages $932 ? 942$.

Bagwell, L. S., and B. D. Bernheim (1996), "Veblen Effects in a Theory of Conspicuous Consumption," American Economic Review vol. 86, pages 349-373.

Balan, D. and G. Deltas (2013), "Better Product at Same Cost, Lower Sales and Lower Welfare," International Journal of Industrial Organization, vol. 31, pages 322-330.

Besanko, D., S. Donnenfeld, and L.J. White (1987), "Monopoly and Quality Distortion: Effects and Remedies," Quarterly Journal of Economics, vol. , pages 743-768.

Bilancini, E. and L. Boncinelli (2008), "Ordinal vs Cardinal Status: Two Examples," Economics Letters vol. 101, pages 17-19.

Boskin, M.J. and E. Sheshinski (1978), "Optimal Redistributive Taxation when Individual Welfare Depends upon Relative Income," Quarterly Journal of Economics vol. 92, pages 589-601.

Bursztyn, L., B. Ferman, S. Fiorin, M. Kanz, and G. Rao (2017), "Status Goods: Experimental Evidence from Platinum Credit Cards," manuscript.

Clark, A.E. and A.J. Oswald (1998), "Comparison-Concave Utility and Following Behavior in Social and Economic Settings," Journal of Public Economics vol. 70, pages 133-155.

Clark, A.E., P. Frijters, and M.A. Shields (2008), "Relative Income, Happiness, and Utility: An Explanation for the Easterlin Paradox and Other Puzzles," Journal of Economic Literature vol. 46, pages $95-144$.

Deltas, G., T. Stengos, and E. Zacharias (2011), "Product Line Pricing in a Vertically Differentiated Oligopoly," Canadian Journal of Economics vol. 44, pages 907-929.

Dupor, B. and W-F Liu (2003), "Jealousy and Equilibrium Overconsumption," American Economic Review vol. 93, pages 423-428.

Ferrer-i-Carbonell, A. (2005), "Income and Well-Being: An Empirical Analysis of the Comparison 
Income Effect," Journal of Public Economics, vol. 89, pages 997-1019.

Frank, R. H. "Positional Externalities," in R.J. Zeckhauser, ed., Strategy and Choice, Cambridge, Mass.: MIT Press, 1991.

Frank, R. H. (2005), "Positional Externalities Cause Large and Preventable Welfare Losses," American Economic Review vol. 95, pages 137-141.

Friedrichsen, J. (2018), "Signals Sell: Product Lines when Consumers Differ Both in Taste for Quality and Image Concern," Discussion Paper No. 70, Collaborative Research Center, U. of Munich.

Gabszewicz, J.J., A. Shaked, J. Sutton, and J.F. Thisse, (1986), "Segmenting the Market: The Monopolist's Optimal Product Mix," Journal of Economic Theory, vol. 39, pages 273-289.

Gabszewicz, J. J., and J-F. Thisse (1979), "Price competition, quality and income disparities," Journal of Economic Theory, vol. 20, pages 340-359.

Ghazzai, H. (2008), "Multi-Product Strategies and Relative Preferences for Quality," Economics Bulletin vol. 4, pages 1-10.

Grilo I., O. Shy, J-F Thisse (2001), "Price competition when consumer behavior is characterized by conformity or vanity," Journal of Public Economics, vol. 80, pages 385-408.

Hopkins, E., and Kornienko, T. (2004), "Running to keep in the same place: consumer choice as a game of status," American Economic Review, vol. 94, pages 1085-1107.

Hopkins, E., and T. Kornienko (2009), "Status, Affluence, and Inequality: Rank-Based Comparisons in Games of Status," Games and Economic Behavior, vol. 67, pages 552-568.

Itoh, M. (1983), "Monopoly, Product Differentiation and Economic Welfare," Journal of Economic Theory, vol. 31, pages 88-104.

Klemperer, P. and A. J. Padilla (1997), "Do Firms' Product Lines Include Too Many Varieties?," RAND Journal of Economics, vol. 28, pages 472-488.

Lambertini, L. and R. Orsini (2002), "Vertically Differentiated Monopoly with a Positional Good," Australian Economic Papers, vol. 41, pages 151-163.

Lambertini, L. and R. Orsini (2005), "Positional Effects, Product Quality and Regulation in Duopoly," Louvain Economic Review, vol. 71, pages 367-381.

Lambertini, L. and R. Orsini (2006), "Multiproduct Monopoly with Positional Externalities," in L. Lambertini, editor, The Economics of Vertically Differentiated Markets, Chapter 2, pages 25-42, Elgar: Cheltenham, UK.

Landis, B. and J. J. Gladstone (2017), "Personality, Income, and Compensatory Consumption: Low-Income Extraverts Spend More on Status," Psychological Science, forthcoming. 
Leibenstein, H. (1950), "Bandwagon, Snob, and Veblen Effects in the Theory of Consumers' Demand," Quarterly Journal of Economics, vol. 64, pages 183-207.

Ljungqvist, L. and H. Uhlig (2000), "Tax Policy and Aggregate Demand Management Under Catching Up with the Joneses," American Economic Review vol. 90, pages 356-366.

Lin, Yuanfang and Chakravarthi Narasimhan (2006), "Persuasive Advertising and Product Line Design," manuscript.

Luttmer, E.F.P. (2005), "Neighbors as Negatives: Relative Earnings and Well-Being," Quarterly Journal of Economics, vol. 120, pages 963-1002.

Mazali, R. and J.A. Rodrigues-Neto (2013), "Dress to Impress: Brands as Status Symbols," Games and Economic Behavior, vol. 82, pages 103-131.

Moldovanu, B., A. Sela, and X. Shi (2007), "Contests for Status," Journal of Political Economy, vol. 115, pages 338-363.

Mussa, M. and S. Rosen (1978), "Monopoly and Product Quality," Journal of Economic Theory, vol. 18, pages 301-317.

Pesendorfer, W. (1995), "Design Innovation and Fashion Cycles," The American Economic Review, vol. 85 , pages 771-792.

Rayo, L. (2013), "Monopolistic Signal Provision," The B.E. Journal of Theoretical Economics, vol. 13, pages 27-58.

Solnick, S.J. and D. Hemenway (1998), "Is More Always Better? A Survey on Positional Concerns," Journal of Economic Behavior and Organization vol. 37, pages 373-383.

Solnick, S.J., and D. Hemenway (2005), "Are Positional Concerns Stronger in Some Domains than in Others," American Economic Review vol. 95, pages 147-151.

Tirole, J., The Theory of Industrial Organization, MIT Press: Cambridge, MA, 1988.

Veblen, T., The Theory of the Leisure Class : An Economic Study of Institutions, Macmillan: New York, London.

Vikander, Nick (2010), "Targeted Advertising and Social Status," manuscript.

Wendner, R. and L.H. Goulder (2008), "Status Effects, Public Goods Provision, and the Excess Burden," Journal of Public Economics, vol. 92, pages 1968-1985. 